\title{
A Central Neural Pathway Controlling Odor Tracking in Drosophila
}

\author{
Gemma Slater, ${ }^{1}$ Peter Levy, ${ }^{1}$ K.L. Andrew Chan, ${ }^{2}$ and ${ }^{\circledR C}$ Camilla Larsen ${ }^{1}$ \\ ${ }^{1}$ Medical Research Council Centre for Developmental Neurobiology, King's College London, New Hunt's House, Guy's Campus, London SE1 1UL, United \\ Kingdom, and 'Institute of Pharmaceutical Science, King's College, London SE1 9NH, United Kingdom
}

Chemotaxis is important for the survival of most animals. How the brain translates sensory input into motor output beyond higher olfactory processing centers is largely unknown. We describe a group of excitatory neurons, termed Odd neurons, which are important for Drosophila larval chemotaxis. Odd neurons receive synaptic input from projection neurons in the calyx of the mushroom body and project axons to the central brain. Functional imaging shows that some of the Odd neurons respond to odor. Larvae in which Odd neurons are silenced are less efficient at odor tracking than controls and sample the odor space more frequently. Larvae in which the excitability of Odd neurons is increased are better at odor intensity discrimination and odor tracking. Thus, the Odd neurons represent a distinct pathway that regulates the sensitivity of the olfactory system to odor concentrations, demonstrating that efficient chemotaxis depends on processing of odor strength downstream of higher olfactory centers.

Key words: behavior; chemotaxis; Drosophila; mushroom body; olfaction

\section{Introduction}

Most animals, including Drosophila melanogaster, detect the magnitude and orientation of odor gradients and direct navigation toward the odor source (chemotaxis). It is not clear whether the processing of odor concentrations during chemotaxis occurs beyond higher olfactory centers in the brain since studies have focused primarily on the first olfactory processing center, the antennal lobe (AL). The Drosophila larva is a particularly attractive model system in which to investigate this question since the architecture of the olfactory system is simple, yet supports robust chemotactic responses.

Drosophila larvae chemotax in response to a variety of odors (Fishilevich et al., 2005; Kreher et al., 2008). Studies have shown that larvae use stereotypical behaviors when navigating an odor gradient. A fall in odor concentration is sensed during runs and triggers a stop in forward motion followed by lateral head swings where the larvae bend the head from side to side. It is thought that this maneuver enables the larvae to measure the difference in odor concentration between two points in space. Frequently, lat-

\footnotetext{
Received June 8, 2014; revised Nov. 5, 2014; accepted Nov. 24, 2014

Author contributions: C.L. designed research; G.S., P.L., K.L.A.C., and C.L. performed research; C.L. analyzed data; C.L. wrote the paper.

This work was supported by grants from the Wellcome Trust and the Royal Society. We thank the Bloomington Stock Center for fly strains and the Developmental Studies Hybridoma Bank for providing antibodies. We also thank Iris Salecker, Mark Frye, Matthew Grubb, and Martin Meyer for comments on the manuscript.

The authors declare no competing financial interests.

This article is freely available online through the J Neurosci Author Open Choice option.

Correspondence should be addressed to Camilla Larsen, MRC Centre for Developmental Neurobiology, New Hunt's House, 4.10, Guy's Campus, King's College London, London SE1 1UL, UK. E-mail: camilla.larsen@kcl.ac.uk. DOI:10.1523/JNEUROSCI.2331-14.2015

Copyright $\odot 2015$ Slater et al.

This is an Open Access article distributed under the terms of the Creative Commons Attribution License (http://creativecommons.org/licenses/by/3.0), which permits unrestricted use, distribution and reproduction in any medium provided that the original work is properly attributed.
}

eral head swings are followed by a turn in the direction of higher odor concentration (Gomez-Marin et al., 2011; Gershow et al., 2012). In addition, larvae also implement continuous biased running, called weathervaning, to control navigational orientation (Gomez-Marin and Louis, 2014).

Larvae detect odorants through olfactory receptor neurons (ORNs; Cobb and Domain, 2000), which project axons to the AL. In the $\mathrm{AL}, \mathrm{ORNs}$ connect with projection neurons (PNs), which send axons to the higher olfactory centers, the mushroom body (MB), and the lateral horn ( $\mathrm{LH})$. The MB is required for learning and memory (Davis, 1993), whereas innate behavior is associated with the LH (Heimbeck et al., 2001). Studies have shown that odor quantity is encoded at the level of the AL, as additional receptors are recruited as odor concentration increases. To prevent stimulus saturation at high odor concentrations, GABAergic interneurons provide gain control within the AL (Asahina et al., 2009). As odor concentration increases, so does the number of ORNs activated, which leads to the recruitment of more inhibitory interneurons and a decrease in gain. This is an important mechanism that prevents larvae from straying too far from a food source where odor concentrations are high.

Since stimulus input is continuously modulated by gain control, how can the larvae respond to low odor concentration during chemotaxis? Here we provide evidence that in the larvae a system operates downstream of the AL that serves to modulate gain by increasing the sensitivity of the olfactory system to low odor concentration. This system is composed of a group of neurons expressing the transcription factor Odd-skipped, termed Odd neurons. These neurons project dendrites into the $\mathrm{MB}$ and axons to the central brain. We provide evidence that the Odd neurons synapse with PNs at the level of the MB calyx. Using functional imaging, we show that a subpopulation of Odd neurons responds to odors. Behavioral assays combined with motion 
tracking show that modulating Odd neuron function affects larval chemotaxis behavior at low odor concentrations but does not abolish odor detection.

\section{Materials and Methods}

Fly genetics. Flies were maintained on a standard molasses medium at $25^{\circ} \mathrm{C}$ on a $12 \mathrm{~h}$ day/night cycle. An equal distribution of males and females were used in all the experiments. Anatomy of the Odd neurons was examined using Odd-Gal4 (Bras-Pereira et al., 2006), to drive expression of the following: UAS-CD8GFP, to label cell morphology (Bloomington Stock Center); UAS-DenMark to label dendrites; andUAS-Synaptotagmin-GFP to mark presynaptic sites (Nicolaï et al., 2010). The expression of neurotransmitters was assessed using the ChatGal4 (Salvaterra and Kitamoto, 2001) to label cholinergic neurons (Bloomington Stock Center), DV-Glut-Gal4 (Daniels et al., 2008) to label glutamatergic neurons, Ddc-Gal4 (Li et al., 2000) to label serotonergic and dopaminergic neurons, Tdc-Gal4 (Cole et al., 2005) to label octopaminergic neurons, and NFP-Gal4 (Johard et al., 2008) to label Neuropeptide F-expressing neurons. For the Mosaic Analysis with a Repressible Cell Marker (MARCM) clones, we used, in addition to the Odd-Gal4 line, FRT 19A, Tub-Gal80ts, and FRT19A, hs-FLP, UAS-CD8GFP (Bloomington Stock Center), and Dynein mutant (Zheng et al., 2008). GFP reconstruction across synaptic partners (GRASP) was performed using, in combination with the Odd-Gal4 driver, GH146-LexA-GAD (Sudhakaran et al., 2012), MB247-LexA-VP16 (Burke et al., 2012), UAS-CD4::spGFP1-10, and LexAop-CD4::spGFP11 (Kreher et al., 2008). For functional imaging UAS-GCaMP6(fast) (Bloomington Stock Center; Akerboom et al., 2012) was driven using the Odd-Gal4 line. LexAopGCaMP6(fast) (Pfeiffer et al., 2010) was driven by the GH146-LexA-GAD line to address PN activity in loss-of-Odd and gain-of-Odd neural function experiments. Odd neural activity was manipulated using the OddGal4 driver in combination with Tub-Gal80ts (Bloomington Stock Center), and UAS-kir2.1 (Cognigni et al., 2011), UAS-NaChBac, UASdORKA-NC (Nitabach et al., 2006), UAS-TNT (Masuda-Nakagawa et al., 2009), or UAS-TrpA1 (Rosenzweig et al., 2005).

Genotypes used in chemotaxis assays. Several genotypes were used in this study, and below we have included a detailed description of how they were used in this study. By including the temperature-controlled Gal4 inhibitor Gal80ts in the genetic crosses, we can limit the expression of neuromodulators to the relevant developmental stage, thereby abolishing any effect on early neurogenesis. In the loss-of-function experiments, we used the two parental strains as controls (Odd-Gal4 and Gal80ts, UAS-Kir2.1) to ensure that any behavioral phenotype observed in the experimental larvae are not due to an unknown background mutation influencing olfaction in either strain. In addition, we also included a third control where, theoretically, the level at which the Odd neurons will be activated is restored by including $\mathrm{NaChBac}$ in the Odd-silenced larvae. This approach has previously been used to rescue behavioral phenotypes induced by kir2.1 expression (Nitabach et al., 2005). Our fourth control is the Orco mutant, which lacks the Orco coreceptor (Benton, 2009), which is required for olfactory receptor function. Larvae lacking this receptor do not show a preference for an attractive odor (Benton, 2009). In the gain-of-function experiments, we used the two parental lines (Odd-Gal4 and Gal80ts, UAS-NaChBac) for reasons similar to those in the loss-of-function experiments. We also included the nonfunctional potassium channel ORK ( $\mathrm{dORK} \Delta-\mathrm{NC})$, since this genetic strain has previously been used as a control for $\mathrm{NaChBac}$ (Nitabach et al., 2005, 2006).

MARCM clones. MARCM clones were generated following the protocol of Lee and Luo (2001). Briefly, eggs were collected on standard yeasted apple juice plates for $1 \mathrm{~h}$ and allowed to develop at $25^{\circ} \mathrm{C}$ until the desired developmental stage followed by heat shock at $37^{\circ} \mathrm{C}$ for $45 \mathrm{~min}$. Eggs were incubated at $25^{\circ} \mathrm{C}$ until second-instar larvae and larvae positive for clones were transferred into vials containing standard molasses medium. Crawling thirdinstar larvae were chosen for analysis of all clonal morphology since these larvae were easier to dissect. However, clonal morphology is similar across all larval stages as the neurons are born during embryogenesis. Dissected larvae were processed for immunocytochemistry as described below. To generate Dynein mutant NB clones, eggs were heat shocked at $3.5 \mathrm{~h}$ after egg laying.
For the MARCM clonal map, eggs were heat shocked at hourly time points from $3.5 \mathrm{~h}$ after egg laying onward.

Immunofluorescence microscopy. Larval brains were dissected in cold PBS and fixed in a $4 \%$ paraformaldehyde/PBS solution for $30 \mathrm{~min}$ at room temperature. The brains were dehydrated in $100 \%$ methanol and stored for at least $24 \mathrm{~h}$ in methanol at $-20^{\circ} \mathrm{C}$ before immunohistochemistry. Brains were rehydrated in PBS containing 0.5\% Triton X-100 (PBT) and blocked in $1 \%$ goat serum (GS) for a minimum of $2 \mathrm{~h}$ before antigen detection with primary antibody overnight at $4{ }^{\circ} \mathrm{C}$ in PBT plus $0.5 \%$ GS. Following primary antibody incubation, brains were washed for $8 \mathrm{~h}$ and incubated overnight in secondary antibody at $4^{\circ} \mathrm{C}$ followed by washing for $1 \mathrm{~d}$. Brains were mounted in PBS and viewed using a Zeiss 510 confocal microscope using either an air $20 \times$ or oil-immersion $40 \times$ or $63 \times$ objectives. The following antibodies were used: mouse monoclonal anti-Bruchpilot (nc82; 1:10 dilution; Developmental Studies Hybridoma Bank), polyclonal rabbit anti-GFP (1:500 dilution; Invitrogen), polyclonal chicken anti- $\beta$-galactosidase (1:1000 dilution; catalog \#ab9361, AbCam), and rabbit anti-GABA (1:100 dilution; catalog \#A2052, Sigma). The secondary antibodies (Invitrogen) used were as follows: Alexa Fluor 488 donkey anti-rabbit, Alexa Fluor 546 donkey anti-chick, and Alexa Fluor 546 goat-anti-mouse. These were used at a 1:500 dilution. For live imaging, larval brains were dissected in cold PBS, mounted, and viewed as for fixed preps.

Functional imaging. Second-instar larvae were wounded on a nitrocellulose-covered glass slide, and the posterior third of the larvae was removed, leaving the anterior body, including the head, intact. The head was inserted into a small hole, and the rest of the body was bathed in a solution containing $5 \mathrm{~mm}$ Na-HEPES (Sigma), pH 7.5, $115 \mathrm{~mm} \mathrm{NaCl}$ (Ambion), $1 \mathrm{~mm} \mathrm{MgCl}_{2}$ (Ambion), $5 \mathrm{~mm}$ KCL (Fisher Scientific), $6 \mathrm{~mm}$ $\mathrm{CaCl}_{2}$ (Sigma), $4 \mathrm{~mm} \mathrm{NaHCO} 3$ (Sigma), $10 \mathrm{~mm}$ glucose (Sigma), and 65 mu sucrose (Alfa Aesar). To minimize larval movement, a coverslip was placed on top of the larvae. The coverslip was held in place by Vaseline applied to each corner. Odor was delivered to the head of the larvae through a steel 27 gauge needle connected to plastic tubing. Air flow was regulated to $40 \mathrm{ml} / \mathrm{min}$ by a mass flow controller (Sable Systems) and delivered via two polytetrafluoroethylene test tubes containing either water or paraffin oil (control) or odorant. Apple cider vinegar (ACV) was diluted $50 \%$ in water to test the responses of the Odd neurons to odor. Neural responses to all monomolecular odors were tested using $10^{-1}$ dilution of odorant in paraffin oil. Two grams of banana mush was used to test Odd neural responses to banana odor. A manually controlled three-way valve system directed air through either test tube. Odor stimuli were delivered for $6 \mathrm{~s}$ with $10 \mathrm{~s}$ intervals. Odor-evoked fluorescent changes were captured using a Zeiss 510 confocal microscope equipped with a $20 \times$ air objective; and imaged at $1 \mathrm{~Hz}$, with image dimensions of $256 \times 256$ pixels, corresponding to a pixel dimension of $1 \mu \mathrm{m}$. Cell bodies [regions of interest (ROIs)] were manually defined from a mean fluorescence image generated from all frames of a time-lapse dataset. Mean normalized fluorescence changes $(\Delta F / F)$ of all voxels within the manually defined region of interest were calculated using customwritten Matlab code. The baseline $(F)$ was calculated for each ROI from the mean fluorescence signal of 10 frames of a time-lapse sequence immediately before odor delivery.

Chemotaxis assays. The standard chemotaxis assay used in our study is designed to test the ability of the larvae to locate a point source of odor within a given time period. To prepare larvae for the chemotaxis assay, $\sim 100$ flies were allowed to lay eggs on yeasted standard apple juice plates for $12 \mathrm{~h}$ at $18^{\circ} \mathrm{C}$. The plates were removed from the cages and incubated for a further $2 \mathrm{~d}$ at $18^{\circ} \mathrm{C}$. Twenty-two hours before testing, the plates were transferred to a $29^{\circ} \mathrm{C}$ incubator to repress Gal80 expression and turn on neural modulators. Controls were raised and tested in similar conditions. Larvae were collected, washed, and transferred onto a $1 \%$ agarose layer in a Petri dish followed by $2 \mathrm{~h}$ starvation. All assays were performed at room temperature except for the $\operatorname{TrpA} 1$, which requires a temperature shift to $30^{\circ} \mathrm{C}$ to ensure full activation of the TrpA1 receptor. For all assays, larvae were sized matched across all genotypes tested to ensure that chemotaxis behavior was not influenced by larval size. To avoid external cues, such as light, affecting larval behavior, we always performed the assays at the same location and always rotated the odor arena $90^{\circ}$ between each exper- 
iment. Approximately 50 second-instar larvae were placed in zone 0 (see Fig. $4 A$ ) on a $1 \%$ agarose gel. Before adding odor, larvae were carefully separated in zone 0 using a fine brush since larvae that are in physical contact with one another travel slower than individual larvae, and this would affect their position within the arena. An appropriate dilution of odorant and control liquid was added to $1 \mathrm{~cm}$ Whatman paper placed directly opposite each other in either zone +4 or zone -4 . We initially placed a $1 \mathrm{~cm}$ plastic disc beneath the Whatman paper to prevent the odor solution from diffusing into the agarose layer. However, we found no difference in behavior between assays performed with and without the plastic disc, and, therefore, we excluded the disc form the assay.

Immediately after adding odor to the arena, a lid was placed on top of the Petri dish, and the larvae were allowed to wander for $5 \mathrm{~min}$. We introduced seven small ventilation holes in the lid to allow excess odor to escape, thereby generating an odor gradient within the odor arena. One ventilation hole was immediately above both the odor source and was above the control. At the end of the experiment, the number of larvae in each zone was calculated. Larvae that did not leave zone 0 were not included in the data collections. These were typically larvae that were damaged during transfer, but extreme care was taken to avoid this, and we found that there were rarely more than three larvae within zone 0 . A response index (RI) was calculated by deducting the number of larvae in a given minus zone from that of the number of larvae in the equivalent plus zone divided by the total number of larvae in the assay. A positive RI shows that larvae can chemotax toward the odor (zone +4 ), whereas an RI of 0 would indicate that larvae have no preference for the odor over the control side. A negative RI shows that larvae are repelled by specific odors. Unless otherwise stated, we used $20 \mu \mathrm{l}$ of ACV as a standard attractive odor and $20 \mu \mathrm{l}$ of water as a control. Monomolecular odors were diluted 1:10 in paraffin oil, and paraffin oil alone was used as the control. Approximately $2 \mathrm{~g}$ of over-ripe banana was used to test behavioral responses to banana mush. The monomolecular odors were chosen based on the following three criteria: (1) they were found to be strongly attractive (Fishilevich et al., 2005); (2) they represent different types of odor groups (i.e., aldehyde, ester, ketone, alcohol, and aromatics); and (3) they bind to a wide range of odorant receptors, thus testing as large a proportion of the neural input into the $\mathrm{AL}$ as possible.

Odor discrimination assay. To test the ability of the larvae to differentiate between two odor concentrations, we built a custom-made Y-shaped plastic arena. Similar to the standard chemotaxis assay, we added a $1 \%$ agarose gel to the bottom of the arena. A 1-cm-diameter piece of Whatman paper was positioned at the distal end of each arm. Approximately 50 larvae were positioned within the bottom $1.5 \mathrm{~cm}$ of the $\mathrm{Y}$ and were separated from each other using a fine brush. Odor was added at the appropriate concentrations to the Whatman paper, a lid with ventilation holes was positioned directly above the odor, and a control was used to cover the assay. We reasoned that as the larvae wander up the odor gradient they have the opportunity to sample both odor concentrations. We tested a 10-fold difference in concentration between the two arms by adding $20 \mu \mathrm{l}$ of ACV at full concentration to one arm and $20 \mu \mathrm{l}$ of a $10^{-1}$ dilution of ACV to the other arm. A fourfold concentration difference was achieved by adding $20 \mu \mathrm{l}$ of ACV at full concentration to one arm and a 1:4 dilution of ACV to the other arm. To achieve a fourfold difference in odor concentration for the monomolecular odors tested, we applied a $10^{-1}$ dilution of the odorant in paraffin oil to one arm and a fourfold dilution of this mixture to the other arm. The larvae were allowed to wander for $10 \mathrm{~min}$, since we found this to be the time window required for most larvae to reach the odor sources in the arms. Larvae that did not enter the arms were not included in data collection. At the end of the experiment the number of larvae was counted in both arms and an RI index was calculated by deducting the number of larvae in the arm containing the lower odor concentration from the number of larvae that reached the arm with the higher concentration of odor. This number was divided by the total number of larvae that entered the arms.

Circular odor discrimination assay. Odor discrimination was also tested in a circular arena similar to that used for the chemotaxis assay. The higher concentration of odor was placed in zone +4 , and the diluted odorant in zone -4 . Larvae were allowed to wander for $5 \mathrm{~min}$, after which the number of larvae were counted in zones $+4,+3,+2,-4,-3$, and -2 . To calculate the RI, the total number of larvae in the negative zones was deducted from the total number of larvae in the positive zones and divided by the total number of larvae (50). These zones were chosen to ensure that larvae had navigated some way toward their chosen odor concentration, thus having made a choice of a preferred odor concentration. All three zones were included since the majority of Odd-silenced larvae do not reach the odor source but do navigate toward it. Similar to the Y maze, we tested the ability of Odd-excited larvae to discriminate between 10 -fold and 4 -fold differences in concentration. In addition, we tested several concentration differences for Odd-silenced larvae and found that at a sixfold concentration difference Odd-silenced larvae cannot discriminate between the two odor concentrations, whereas the controls can. This concentration difference was therefore chosen to compare odor discrimination between the Odd-silenced larvae and controls.

Hanging drop assay. The odor arena used in the hanging drop assay was similar to that of the chemotaxis assay except that $20 \mu \mathrm{l}$ of ACV was added to the middle of the lid. Single larvae were placed directly beneath the odor source and allowed to wander for $5 \mathrm{~min}$. The position of larvae was manually scored at $15 \mathrm{~s}$ intervals. This time window was used because we found that larvae would spend a minimum of $15 \mathrm{~s}$ within each ring and this therefore allowed us to score the position within all the rings in which the larvae navigated. Six ventilation holes were situated equidistantly $\sim 1 \mathrm{~cm}$ from the edge of the lid. In addition, we divided the lid into $0.25 \mathrm{~cm}$ concentric rings to score the larval position in relation to the odor source.

Live imaging and locomotion tracks. We imaged larval behavior during both the chemotaxis and the hanging drop assay. Larvae were imaged using a Nikon SMZ1500 dissecting microscope with ambient light at room temperature. Movies were captured on a Nikon Digital sight DSRil camera at a frame rate of 7 frames/s. FIMtrack software (Risse et al., 2013) was used to analyze turning behavior during the chemotaxis assay. As part of the analysis, FIMtrack generates full locomotion tracks of all the larvae that were analyzed. We combined the locomotion tracks from all the analyzed larvae in CorelDRAW X3 (Corel) and manually traced over the tracks. Using FIJI64 (ImageJ) a Z-stack projection was generated from each movie filmed during the hanging drop assay. These were used in CoreIDRAW X3 to manually trace the position of the larvae throughout the duration of the entire experiment.

Statistical and behavioral analyses. GraphPad Prism5 was used for all statistical analyses. Each chemotaxis assay was repeated 10 times, and statistical significance was calculated using a two-way ANOVA test and Bonferroni post-test. In the hanging drop assay $(N=15)$, the position of larvae was manually scored every $15 \mathrm{~s}$ and normalized to the time spent in each circle calculated. A Wilcoxon test corrected for multiple comparisons with a confidence interval of $95 \%$ was used to evaluate statistical significance between experimental larvae and controls. Time spent in each circle was compared separately, and statistically significant differences between controls and experimental larvae were calculated. Multiworm tracker (MWT) software was used to measure the speed of locomotion $(N=15)$. Velocity values generated by MWT were directly plotted in GraphPad Prism5 to calculate the average speed of the larvae. We used a Kruskal-Wallis test combined with a Bonferroni post-test to analyze statistical significance among the average speeds of the genotypes tested. FIMTrack was used to analyze the frequency of larval body bending, stops, and turns in the chemotaxis assay both in the presence and absence of odor. We preset the angle to $60^{\circ}$ in the software so that body bending at a lower degree was not included in the analysis. An angle of $60^{\circ}$ was chosen since, during the run phase, larvae were seen to carry out head swings that are not associated with directional turning. The vast majority of these head swing were $<60^{\circ}$. Since we wanted to analyze head swings associated with directional turning, we therefore excluded these smallamplitude head swings by setting the angle at $60^{\circ}$. FIMTrack assigns a value of 1 if the body angle is $60^{\circ}$, whereas a body angle $<60^{\circ}$ is assigned a value of 0 . The angle of the larvae was scored every $1 \mathrm{~s}$ through the duration of the movie. In addition, left and right bending is analyzed separately by the software, and double head sweeps can be scored if a left or right body bend is followed immediately by a bend in the opposite direction. The number of single and double head swings was plotted in GraphPad Prism5 as a function of head swings per minute. FIMTrack 
assigns a go phase and a stop phase to larval locomotion, and we used these values to plot the number of stops per minute. In addition, FIMTrack continuously measures the angle of forward motion. To analyze turning, we scored abrupt changes in the direction of forward motion and plotted these values against time. We used a Kruskal-Wallis test combined with a Bonferroni post-test to analyze statistical significance among the genotypes tested.

Fourier transform infrared spectroscopic measurements of odor concentration. To ensure that a gradient of odorant is generated in the chemotaxis, hanging drop and circular odor discrimination arenas we measured the concentration of odorant in gas phase using Fourier transform infrared (FTIR) spectroscopy. Odor concentrations were measured in gas phase at the following three points in the arena: in zone +4 , next to the Whatman paper containing the higher concentration of odor; in zone 0 , in the middle of the plate; and in zone -4 next to the Whatman paper containing either control solution or diluted odorant. Circular holes were cut into the base and lid of the arena at each position to allow the infrared beam to measure odor concentrations inside the arena. A beam length of $1 \mathrm{~cm}$ was maintained at all three positions. To maintain odor concentrations similar to that used during behavior experiments, the holes were covered in infrared transparent discs ( made of $\mathrm{CaF}_{2}$ ), and the amount of odorant used was similar to those in the behavior assays. We measured the concentration of isoamyl acetate since this odorant produces an easily distinguishable peak at $2952 \mathrm{~cm}$, which made comparisons between different peak heights more reliable. Similar results were obtained with 2-heptanone. FTIR spectra were measured using a Frontier spectrometer (PerkinElmer) and Spectrum 10 software. The spectral resolution was $4 \mathrm{~cm}$, and scanning time was $5 \mathrm{~min}$, which is comparable to the length of the behavior assays.

\section{Results}

The Odd neurons project dendrites into the calyx of the MB

At larval stages, Odd-skipped is expressed exclusively in eight neurons in the brain, and we have previously shown that the Odd neurons innervate the calyx of the $\mathrm{MB}$ in the larva and the adult (Larsen et al., 2006; Levy and Larsen, 2013). However, the complete projection patterns and dendritic and axonal regions of these neurons have not been documented. To label Odd neurons, we used the Odd-skipped specific driver line Odd-Gal4 (BrasPereira et al., 2006) in combination with UAS-CD2-GFP. A dorsal view shows that, in addition to extending into the calyx (Fig. $1 A$ ), the Odd neurons also project ipsilaterally and contralaterally, arborizing in the centroposterior medial compartment (CPM; Pereanu et al., 2010; Fig. 1A). They also send a few processes posteriorly. A lateral view (Fig. $1 B$ ) shows that the Odd neurons initially project ventrally then bifurcate, sending projections dorsally into the calyx and ventrally into the CPM. There are also some smaller branches at the point of bifurcation. The CPM arbor and posterior projecting branches do not enter the ventral nerve cord (dotted line), which indicates that Odd neurons are unlikely to synapse with motor neurons. We addressed whether Odd neurons are excitatory or inhibitory by colabeling with either reporter lines of, or antibodies to, different neurotransmitters. To colabel Odd neurons with neurotransmitter Gal4 reporter lines, we took advantage of the Odd insertion line

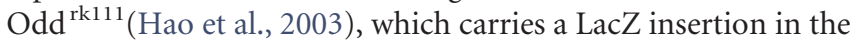
Odd locus. This allows us to detect Odd neurons with a $\beta$-gal antibody while labeling reporter line expression with a GFP antibody. We have previously shown (Levy and Larsen, 2013) that this line recapitulates the expression pattern of Odd-skipped. We tested for the expression of acetylcholine (Fig. 1C,D), octopamine (Fig. $1 E$ ), dopamine, serotonin (Fig. $1 F$ ), glutamate (Fig. $1 G$ ), Neuropeptide F (Fig. $1 H$ ), and GABA (Fig. 1I); and found that Odd neurons are exclusively cholinergic, indicating that they are excitatory.
Next, we mapped the dendritic and axonal compartments of the Odd neural arbor to gain a better picture of the synaptic input to and output from the Odd neurons. Using the dendritic marker Denmark (Nicolaï et al., 2010), which is expressed in the Odd neurons using the Odd-Gal4 line, we show that the projections into the calyx are dendritic (Fig. 1J). Some dendrites are also located at the bifurcation point, whereas the projections into the CPM are axonal (Fig. $1 J$ ), which is shown by localization of the axonal marker synaptotagmin-GFP (Zhang et al., 2002). We confirmed that the projections into the calyx were indeed dendritic by generating Dynein mutant clones using the MARCM technique (Lee and Luo, 2001). Dynein is required for polarized dendritic transport, and the dendritic arbors of dynein mutant neurons are shifted proximally (Zheng et al., 2008). In Odd $d y$ nein mutant clones, dendrites are absent from the calyx (Fig. $1 \mathrm{~K}$ ), whereas a wild-type clone maintains dendritic projections into the calyx (Fig. 1L). To examine whether all eight Odd neurons project dendrites into the calyx, we used MARCM to generate a full clonal map of all the larval Odd neurons. We took advantage of the fact that birth dates of neurons in the CNS are separated by $1 \mathrm{~h}$ to selectively label individual members of the Odd cluster. Single-cell clones could be labeled with GFP from $3.5 \mathrm{~h}$ after egg laying at different time points through embryonic development. At the start of neurogenesis, a single Odd neuron was generated per hour, but from $5.5 \mathrm{~h}$ after egg laying Odd neurons were labeled every second hour of heat shock induction. Odd neural morphology was identical for each tested animal $(N=5)$. Using this approach, we found that only three of the neurons projected into the calyx, whereas the rest extend either ipsilaterally or contralaterally (Figs. $1 M, N, 2$ ). A map generated from the neural tracing of all the single-cell clones (Fig. $1 N$ ) shows the arborization point of individual neurons. All neurons except one (Fig. 2B) arborize in the CPM. Two of the Odd neurons that project into the calyx extend to the contralateral side (Figs. $1 L$, Fig. $2 C$ ), whereas the third neuron projects ipsilaterally only (Fig. 2D). All three neurons that project into the calyx also arborize in the CPM. Our lineage mapping shows that a subset of the Odd neurons projects into the calyx and suggests that Odd neurons could relay input from the olfactory system to both brain hemispheres.

\section{Odd neurons are postsynaptic to PNs in the calyx and respond to odor}

The dendrites of the Odd neurons are positioned within the dendritic compartment (calyx) of the MB Kenyon cells, and this raises the possibility that Odd neurons could receive input from PNs as well as form synapses with the Kenyon cells. To test this, we used GRASP (Gordon and Scott, 2009). One part of the split GFP system (spGFP1-10) was expressed in PNs using the GH146lexA driver, whereas the other part (spGFP11) was expressed in the Odd neurons using the Odd-Gal4 driver. We found that expressing both results in the formation of punctate accumulations of GFP fluorescence in the calyx (90 puncta on average per calyx; $N=6$; Fig. $3 A$ ), whereas expression of either half of the split GFP alone did not result in any visible GFP fluorescence (data not shown). This suggests that the two neural populations are sufficiently close to reconstitute GFP and that the Odd neurons could make synaptic contacts with PNs. In addition, we also tested whether reconstitution of GFP is possible between Kenyon cells and Odd neurons since dendro-dendritic synapses have been reported in flies (Haag and Borst, 2002). We expressed the spGFP1-10 in the Kenyon cells using the Mb247LexA driver in combination with spGFP11 expression in the Odd neurons using the Odd-Gal4 driver. We found that we could indeed observe GFP puncta, but these were fewer in number (on average 21 per 

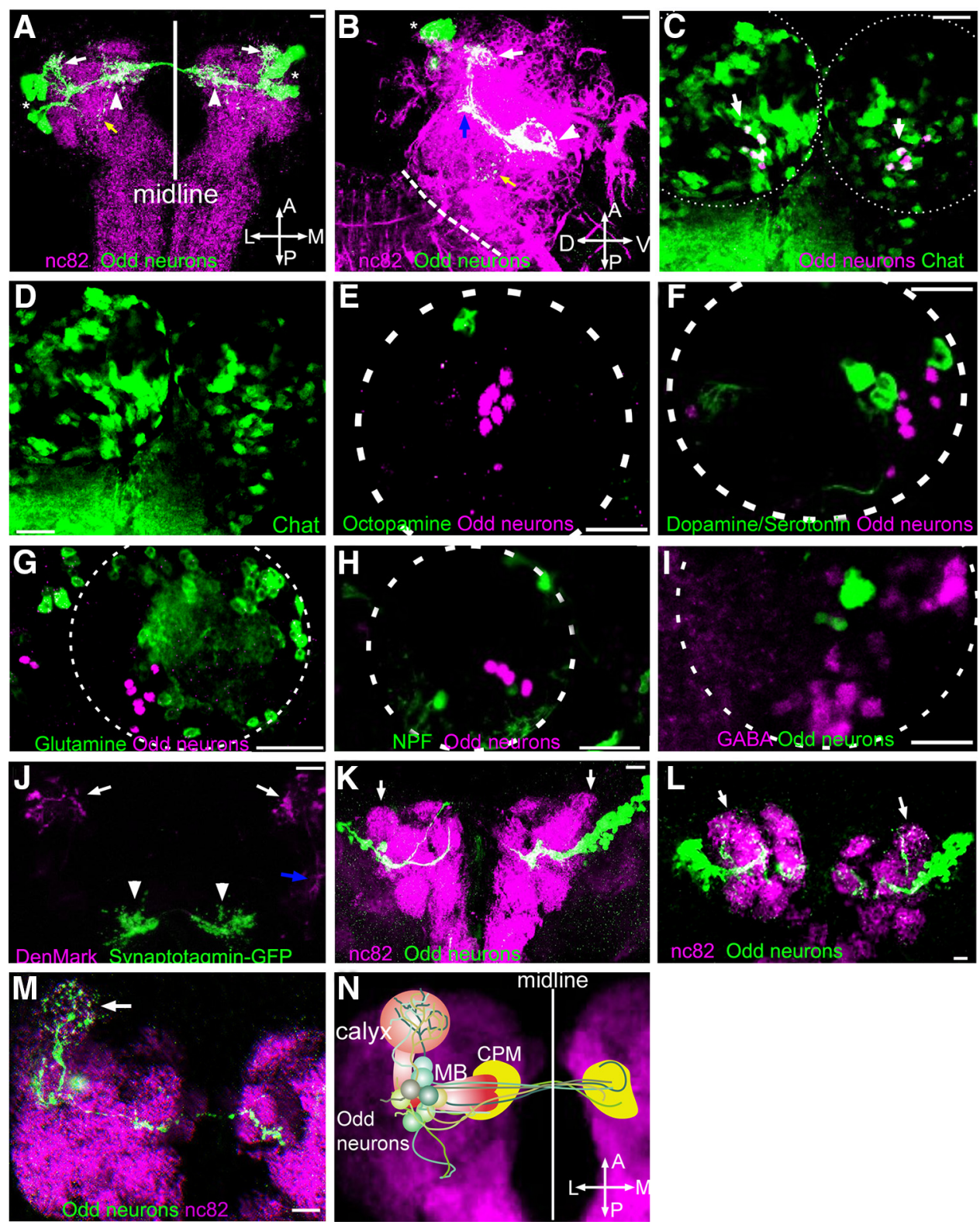

Figure 1. Odd neurons project dendrites to the calyx of the MB. All images show second instar larval brains apart from $M$ and $N$, which are third instar brains. $A, B$, and $J-M$ are maximum intensity confocal stacks. $C-I$ are single confocal sections. $A$, Anatomy of the 0 dd neurons (green) with nc 82 (magenta) as a neuropile marker (dorsal view). 0 dd neurons $\left({ }^{*}\right.$ ) project to the calyx (arrow) and arborize medially in the CPM (arrowhead). There are also a few branches extending posteriorly (yellow arrow). B, Lateral view of a projection pattern showing that the (PM arbor (arrowhead) of the Odd neurons (white, asterisks) does not project into the ventral nerve cord (anterior limit dotted line). The arbor in the calyx (arrow) is located anterior to the cell bodies (asterisks), and a small arbor is located at the point of bifurcation (blue arrow). Also visible are the branches that extend posteriorly (yellow arrow). C, Colocalization between an acetylcholine transferase driver line (green) and Odd neurons (white; arrows). All Odd neurons colocalize with the driver line in both hemispheres (each hemisphere marked by dotted lines). $\boldsymbol{D}$, Green channel only of $\boldsymbol{C}$. $\boldsymbol{E}-\boldsymbol{I}, 0 \mathrm{dd}$ neurons do not colocalize with the Tdc driver line expressed in octopaminergic neurons $(\boldsymbol{E})$, the Ddc driver line expressed in dopaminergic and serotonergic neurons $(\boldsymbol{F})$, the DVGlut-Gal4 line driving expression in glutamatergic neurons $(\boldsymbol{G})$, the NPF driver line expressed in Neuropeptide F-producing neurons $(\boldsymbol{H})$, and GABA antibody expression $(\boldsymbol{I})$. $\boldsymbol{J}$, Driving the dendtirtic marker DenMark (magenta) and the presynaptic marker synaptotagmin-GFP (green) in the 0dd neurons. DenMark localizes to the calyx (arrow) and small arbor at the bifurcation point (blue arrow). Synaptotagmin-GFP localizes to the (PM arbor exclusively (arrowhead). $\boldsymbol{K}$, Dynein mutant 0dd NB clone (green) showing the absence of Odd neural projections in the calyx (arrows). nc82 labels the neuropile (magenta). $L$, Wild-type Odd NB clone (green) showing projections into the calyx (arrows). nc82 labels the neuropile (magenta). $\boldsymbol{M}$, Single-cell wild-type Odd clone (green) of one of the $0 \mathrm{dd}$ neurons that extends projections into the calyx (arrow) and contralaterally into the (PM. This neuron was born $5.5 \mathrm{~h}$ after egg laying. $\mathrm{nc} 82$ labels the neuropile (magenta). $\boldsymbol{N}$, Reconstruction of all eight neurons implemented into a standard larval brain in relation to the MB (red) and (PM (yellow). Scale bars, $20 \mu \mathrm{m}$. A, anterior; P, posterior; D, dorsal; V, ventral; L, lateral; M, medial.

calyx; $N=6$ ) compared with the contacts between the Odd neurons and PNs (Fig. 3B).

We next analyzed the distribution of GRASP puncta between the Odd neurons and PNs to address whether potential synaptic inputs from the PNs are restricted to specific glomeruli. We colabeled brains in which the spGFP was expressed in PNs and Odd neurons with the Bruchpilot-specific antibody (nc82), which labels presynaptic sites. However, we found an equal distribution of puncta across the calyx (Fig. 3C). PN arborizations in the calyx form distinct glomeruli, and individual PNs typically project to one or two individual glomeruli (Ramaekers et al., 2005). Our clonal map shows that the arbor from individual Odd neurons extends throughout the calyx. Thus, individual Odd neurons are likely to receive input from multiple PNs. To confirm that the GRASP puncta represent synapses, we imaged these brains at high resolution and found that the GRASP puncta colocalize with 

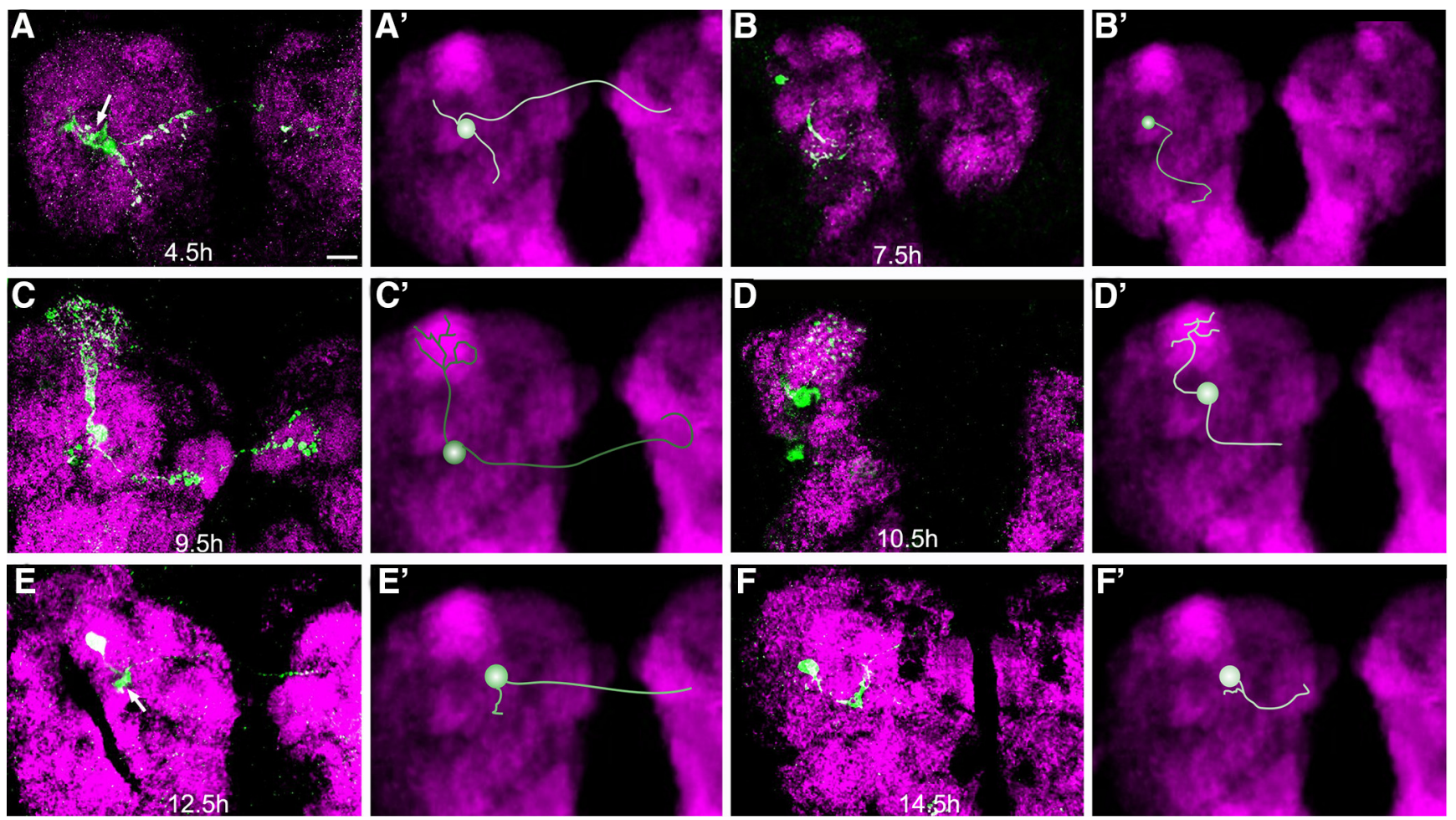

Figure 2. Anatomy of individual Odd neurons. All images are dorsal views of third instar larval brains stained for nc82 (magenta), anterior at the top, lateral to the left. Single MARCM clones (green) induced at different developmental stages. $\boldsymbol{A}^{\prime}-\boldsymbol{F}^{\prime}$ are neural reconstruction implemented in a standard larval brain of each image. $\boldsymbol{A}, \mathrm{Clonal}$ induction at $4.5 \mathrm{~h}$ after egg laying, which labels an Odd neuron that projects contralateral and ipsilateral arborizing near the CPM (arrow). $\boldsymbol{B}$, Heat shock induction at $7.5 \mathrm{~h}$ after egg laying labeling a cell that projects only ipsilateral. $\boldsymbol{C}$, Heat shock induction at $9.5 \mathrm{~h}$ which labels one of the $0 \mathrm{dd}$ neurons that project into the calyx and contralaterally to the CPM. D, Heat shock induction at $10.5 \mathrm{~h}$ after egg laying labeling the third $0 \mathrm{dd}$ neuron that projects into the calyx. This cell exclusively projects ipsilaterally. $\boldsymbol{E}$, Heat shock induction at $12.5 \mathrm{~h}$ after egg laying. This $0 \mathrm{dd}$ neuron has a small arbor close to the cell body (arrow) and projects contralaterally. $\boldsymbol{F}$, Heat shock induction at $14.5 \mathrm{~h}$ after egg laying labeling an ipsilateral projecting cell.

the presynaptic marker Bruchpilot (Fig. 3D), suggesting that they represent synaptic connections between Odd neurons and PNs.

If the Odd neurons receive synaptic input from PNs, we would expect that they respond to odor. We tested this by expressing GCaMP6(fast) (Chen et al., 2013) exclusively in the Odd neurons and imaged neural responses to the attractive odor ACV. Odors were delivered through a continuous air stream for multiple cycles lasting 6 seconds each. We could repeatedly generate odorevoked responses in a subset of the Odd neurons (Fig. $3 E-E^{\prime \prime}, F$; $N=9$ ). These data demonstrate that some Odd neurons respond to odor and are likely to receive odor information via synaptic input from the PNs. We next asked whether Odd neurons respond similarly to different concentrations of ACV. We found a decrease in responses when larvae were exposed to a 10-fold dilution of ACV (Fig. 3G,H). Further lowering the odor concentration to a 1:20 dilution of ACV does not significantly change the average peak response $(N=6)$ compared with a 1:10 dilution (Fig. $3 H$ ). These data show that Odd neurons can respond to low odor concentrations.

Our anatomical data show that the Odd neurons project axons to the CPM and that the Odd neurons are therefore unlikely to provide direct feedback onto ORNs or PNs. However, we cannot exclude the possibility that Odd neurons could provide indirect feedback to the PNs. To address this, we imaged PN neural responses in larvae where we either silenced the Odd neurons using Kir2.1, or made them more excitable by expressing the lowactivation threshold, voltage-gated sodium channel ( $\mathrm{NaChBac}$ ) using the Odd-Gal4 driver. Since PNs are the only output from the AL to higher olfactory centers, we reasoned that feedback onto any of the neural components presynaptically to PNs should result in a change to PN activity. Thus, if the Odd neurons form part of a feedback circuit, the resulting changes in neural modulation within the first olfactory center should ultimately lead to a change in PN output. To allow for simultaneous expression of GCaMP6(fast) in the PNs and neural modulators in the Odd neurons, we took advantage of the LexA system to express LexAop-GCaMP6(fast) using the GH146lexA driver. First, we measured the odor-evoked response of PNs to $50 \% \mathrm{ACV}$ in larvae with no modulation of Odd neural activity and found that PNs like the Odd neurons respond to repeated cycles of odor stimuli (Fig. 3I). Unlike the Odd neurons, though, the PN responses were maintained only during odor stimuli, whereas responses are maintained in the Odd neurons for up to $4 \mathrm{~s}$ after odor stimuli are terminated. PN average peak responses did not significantly change in larvae where Odd neural function was modulated (Fig. $3 J$ ), showing that Odd neurons do not provide feedback onto the olfactory system.

\section{Odd neurons are involved in chemotaxis}

Since some of the Odd neurons connect with the olfactory system, we tested their role in chemotaxis using standard larval chemotaxis assays (Kreher et al., 2008). To allow for better spatial resolution of larval behavior, we adopted the approach of Fishilevich et al. (2005) and divided a $9 \mathrm{~cm}$ Petri dish into $9 \times 1$ $\mathrm{cm}$ zones, placing the odor source in zone +4 and either water or paraffin oil as a control in zone -4 , depending on which liquid was used to dilute the odor (Fig. $4 A$ ). Larvae were placed in zone 0 , halfway between zone +4 and zone -4 , and allowed to wander for $5 \mathrm{~min}$, after which the number of larvae in each zone was counted. An RI for each zone is calculated (see Materials and 

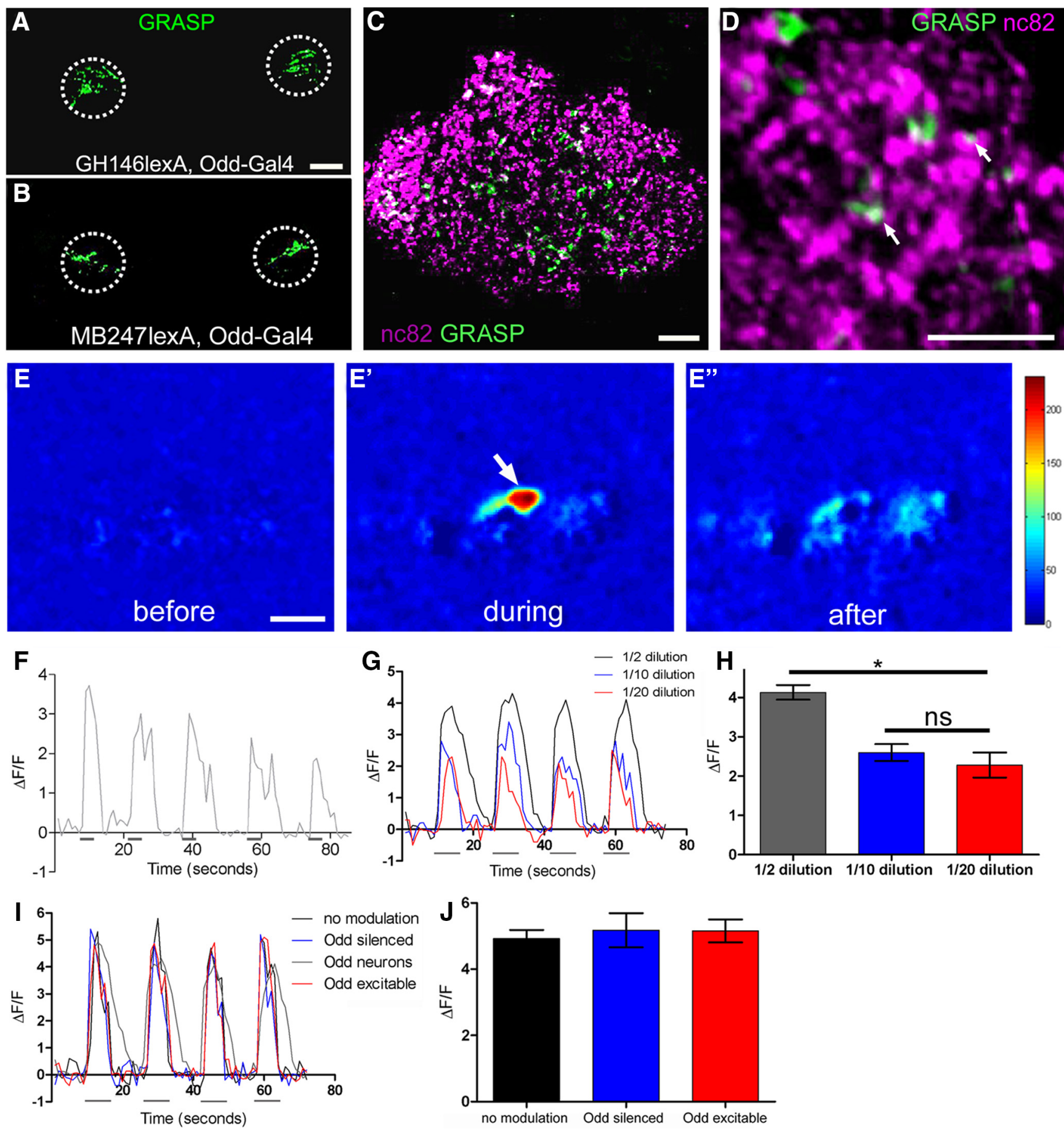

Figure 3. Odd neurons receive input from PNs and respond to odor. All images are second instar larval brains. A, Confocal stack of a larval brain expressing the split-GFP (GRASP; green) in PNs (GH146lexA) and Odd neurons (Odd-gal4). Reconstitution of GFP is restricted to the calyx (dotted circles). B, Confocal stack of a larval brain expressing the split-GFP in Kenyon cells (MB247lexA driver) and Odd neurons (Odd-gal4 driver). A few GRASP puncta localize to the calyx (dotted circle). C, Confocal stack, high-magnification (63X) image of the calyx showing split-GFP localization between the PNs and Odd neurons (green) colabeled with nc82, which labels presynaptic densities. GRASP puncta are distributed throughout most of the calyx. D, Single confocal image $(63 \times, 5 \times$ zoom) of the calyx showing split-GFP localization between the PNs and Odd neurons (green) in the background of nc82 staining labeling presynaptic sites. Part of the GRASP puncta colocalizes with nc82 (examples labeled by arrows), showing that these puncta are likely synapses. $\boldsymbol{E}$, Response of Odd neurons expressing GCaMP6 to ACV. $\boldsymbol{E}$, Before odor stimuli. $\boldsymbol{E}^{\prime}$, During odor stimuli. $\boldsymbol{E}^{\prime \prime}$, After odor stimuli. Some cells respond to odor (arrow). $\boldsymbol{F}$, Response of a single cell to repeated cycles of odor presentation. Gray boxes represent odor stimuli. G,Dose-response curve showing the response profile of individual Odd neurons to different concentrations of ACV. Gray boxes represent odor stimuli. $\boldsymbol{H}$, Lowering the concentration 10-fold significantly decreases the average maximum responses of the $0 \mathrm{dd}$ neurons to ACV, (Kruskal-Wallis test, Bonferroni post-test, $p<0.05$ ) but responses are similar between 1:10 and 1:20 dilutions of ACV.I, Response profile of PNs in larvae in which the Odd neurons are either silenced using Kir2.1 or made more excitable using NaChBac. Gray boxes represent odor stimuli. J, Neither manipulation affects the average maximum response of PNs (Kruskal-Wallis test, Bonferroni post-test). Scale bars: $A, B, F-I, 20 \mu \mathrm{m} ; C, E, 10 \mu \mathrm{m} ; D, 5 \mu \mathrm{m}$.

Methods). This approach allows us to quantify the proportion of larvae that navigate toward the odor source placed in zone +4 as well as to assess the number of larvae that reach the odor source. For these numbers to be accurate, larvae that reach the odor source in zone +4 during the assay should stay on the odor source and not wander back into zone +3 and +2 , which would give the impression that these larvae have not reached the odor source by the end of the assay. We therefore filmed larvae during 

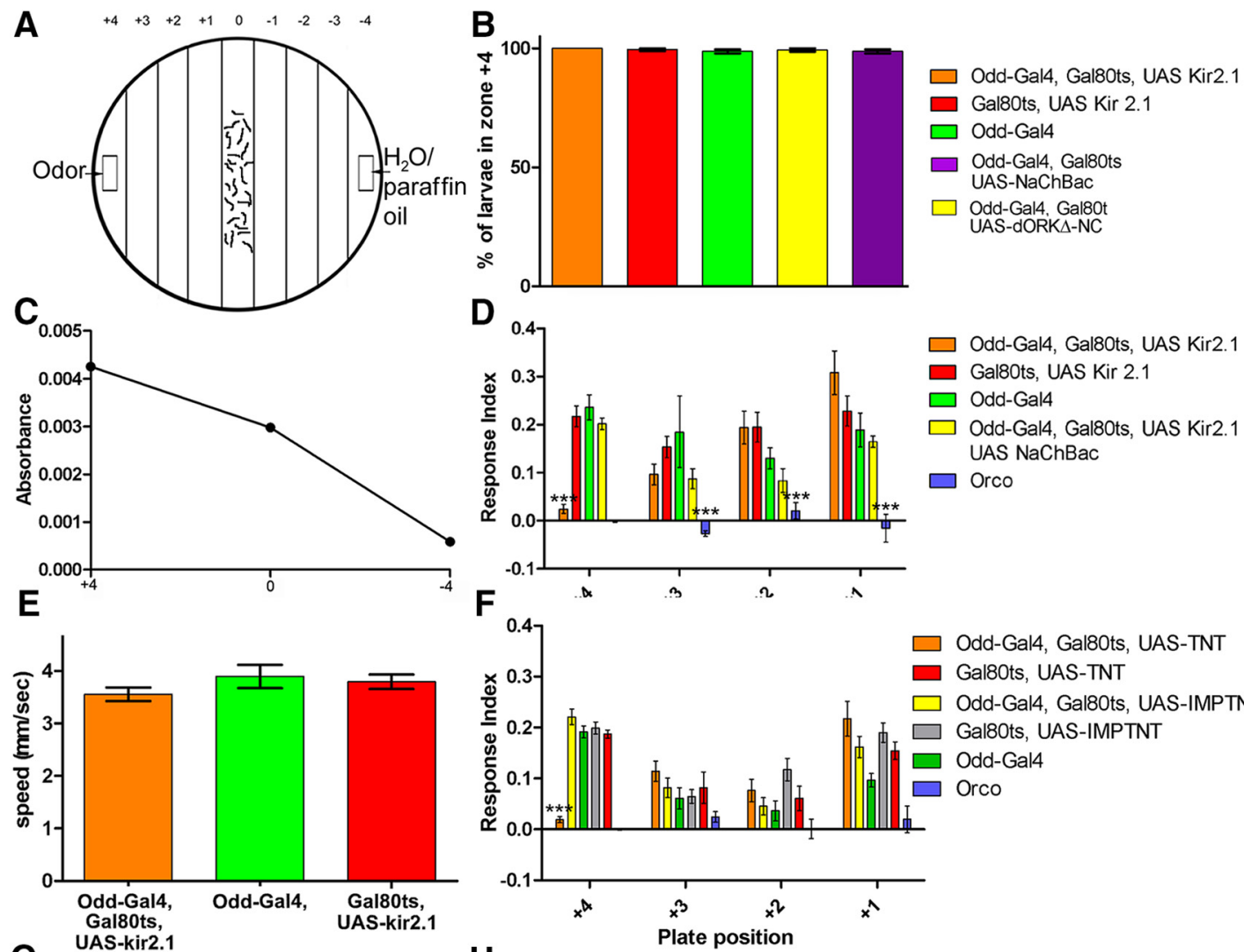

F
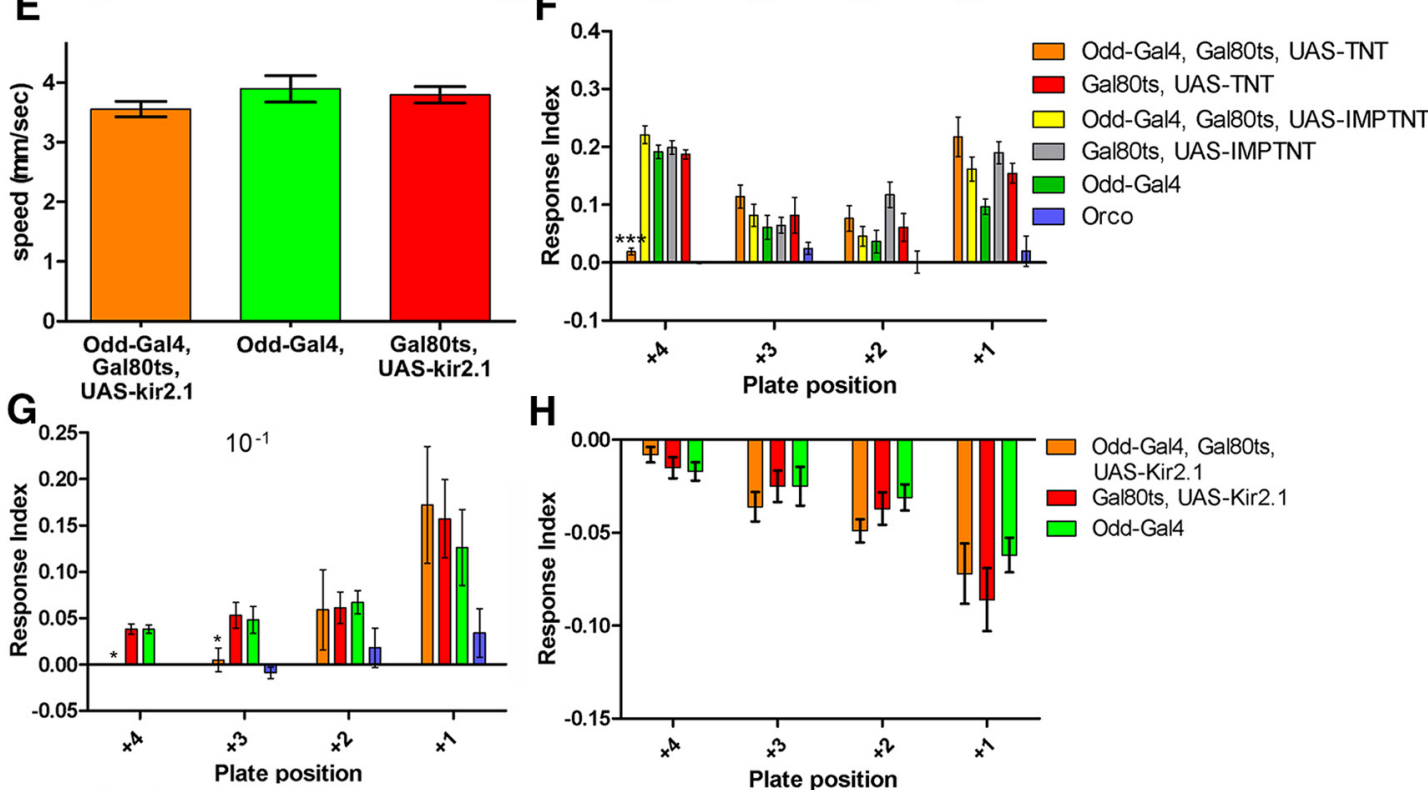

$\square$ Odd-Gal4, Gal80ts, UAS Kir2.1

$\square$ Gal80ts, UAS Kir 2.1

$\square$ Odd-Gal4

$\square$ Odd-Gal4, Gal80ts, UAS Kir2.1

UAS NaChBac

$\square$ Orco

Odd-Gal4, Gal80ts,

$\square$ Odd-Gal4

UAS-Kir2.1

$\square$ Gal80ts, UAS-Kir2.1 $\square$ Orco

I
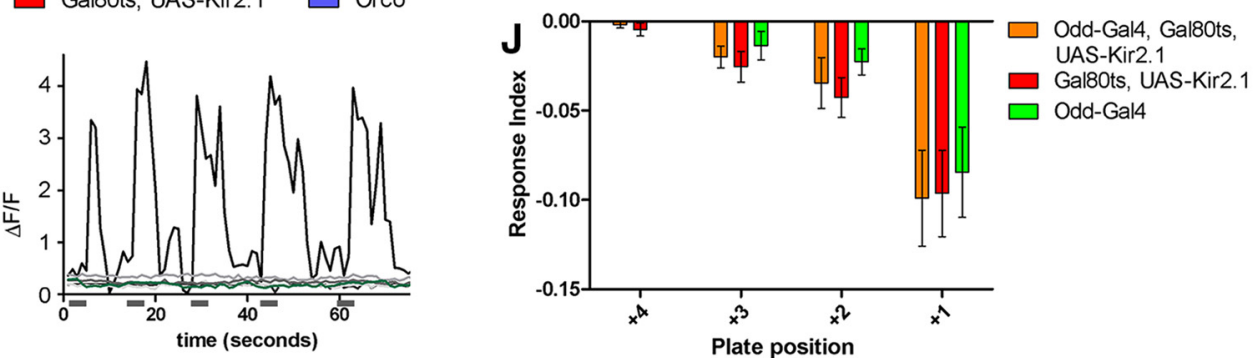

Figure 4. Silencing Odd neurons impairs chemotaxis. $\boldsymbol{A}$, Schematic representation of the chemotaxis behavior assay showing the labeling of each zone at the top. $\boldsymbol{B}$, Percentage of larvae that remain on the odor source during the time period of the chemotaxis assay. Between $100 \%$ and $96 \%$ of all larvae that reach the odor source remain on it until the end of the assay. $C$, Fourier transform infrared spectroscopic measurements of the odor concentrations in gas phase at the following three locations in the chemotaxis arena: near the odor source (zone +4$)$, in the middle (zone 0$)$, and near the control side (zone -4$)$. Odor concentration is highest near the odor source $(z o n e+4)$ and lowest at the control side, showing that a gradient of odor concentrations is generated within the assay. $\boldsymbol{D}$, Behavioral responses to the full concentration of ACV. Odor-evoked behavior is statistically significant for Odd-silenced larvae using Kir2.1 (orange) in zone +4 compared with controls. Odd-silenced larvae behave significantly better than the anosmic Orco control in zones $+3,+2$, and +1 , showing that they do respond to an attractive odor. The RI response is calculated for each zone of the plate ( $x$-axis). Each bar represents the RI \pm SEM; $n=10 .{ }^{*} p<0.05,{ }^{* * *} p<0.001$. E, Locomotion of larvae in the absence of odor calculated from the speed tracks generated by MWT. Each bar represents average locomotion over a $4 \mathrm{~min}$ period ( $y$-axis $) \pm \operatorname{SEM}(n=7)$. $F$, Behavioral responses to full concentration of ACV using TNT to silence the Odd neurons (orange). A similar pattern of behavior is observed as with Kir2.1. G, Behavioral response to $10^{-1}$ dilution of ACV. Odor-evoked behavior is significantly different for $0 \mathrm{dd}$-silenced larvae in zones +4 and +3 . $\boldsymbol{H}$, Larval behavioral responses to the repellent 1-nonanol. There is no significant behavioral difference between Odd-silenced larvae and controls. I, Odd neural responses to 1-nonanol using functional imaging. None of the larvae $(N=5)$ respond to this repellent (traces of different shades of gray) compared with the black trace, which is an example of a response to ACV. Gray boxes represent odor stimuli. J, Larval behavioral responses to the repellent geranyl acetate. There is no significant behavioral difference between Odd-silenced larvae and controls. Statistical significance for all the chemotaxis assays was calculated using a two-way ANOVA and Bonferroni post-test to calculate the degree of statistical significance. 
the chemotaxis assay, focusing on the odor source, and counted the number of larvae that left the odor source before the end of the assay. We found that between $96 \%$ and $100 \%$ of all larvae stay on the odor source once they have reached it (Fig. 4B). This demonstrates that larvae stay on the odor source once they have reached zone +4 , and therefore larvae counted in other zones must be in the process of navigating toward the odor source. To validate that an odor gradient is generated in the chemotaxis arena, we measured the concentration of odorant in the gas phase at the following three locations within the arena: zones $+4,0$, and -4 . We find that a gradient is generated within the chemotaxis assay (Fig. 4C) and that the odor concentration is lower at the starting point of the assay (zone 0 ) compared with near the odor source in zone +4 .

We first asked whether larval chemotaxis is affected by silencing the Odd neurons (Odd-silenced larvae) using the inward rectifier $\mathrm{K}^{+}$channel Kir2.1 (Baines et al., 2001) driven by the Odd-Gal4-specific driver (for detailed description of genotypes used, see Materials and Methods). We found that the proportion of Odd-silenced larvae in zone +4 is significantly lower $(p<$ 0.001, two-way ANOVA) compared with the parental controls (Gal80ts, UAS-Kir2.1, and Odd-Gal4) and larvae in which Odd neural activity is rescued by coexpressing $\mathrm{NaChBac}$ with Kir2.1 (Odd-Gal4, Gal80ts, UAS-Kir2.1, and UAS-NaChBac; Fig. 4D). This shows that silencing Odd neurons impairs chemotaxis. The fact that the larvae in which Odd neural activity has been rescued have an RI that is statistically similar to those of the two parental controls shows that Kir2.1-induced silencing of the Odd neurons is specific to Kir2.1 modulation of neural activity. The Oddsilenced larvae have a positive RI for zones $+3,+2$, and +1 , and, with respect to these zones, are not significantly different from the control groups. However, the Odd-silenced larvae perform significantly better than the anosmic Orco mutant (Orco) larvae $(p<0.001)$, which shows that larvae in which the Odd neurons have been silenced respond to odor and are not anosmic.

The chemotaxis phenotype observed with the Odd-silenced larvae could be due to impaired motor function, and we therefore tested locomotion by filming crawling larvae in the chemotaxis assay arena in the absence of odor. The movies were analyzed using the MWT software (Baek et al., 2002). We found no significant difference in average speed between Odd-silenced larvae and the two parental strains (two-way ANOVA; Fig. 4E), indicating that the failure of Odd-silenced larvae to reach the odor source is not due to a decrease in locomotion speed.

Odd-skipped is also expressed in a group of insulin-producing neurons at the distal portion of the nerve cord. These neurons innervate the intestine and are thought to regulate appetite (Cognigni et al., 2011). We can therefore not rule out that the phenotype observed in the chemotaxis assay could be a result of manipulating appetite. To exclude this possibility, we used an alternative method for silencing the Odd neurons by expressing tetanus toxins (TNTs) since insulin-producing neurons are not silenced by TNTs (Cognigni et al., 2011). We obtained a result with a TNT similar to that with Kir2.1 $(p<0.001$, two-way ANOVA; Fig. $4 F)$. Both the parental strains and a nonfunctional TNT (Odd-Gal4 UAS-IMPTNT) show normal attractive behavior, and we can thus conclude that the phenotype observed with Kir2.1 is not due to manipulating the brain-gut insulin circuit. In addition, using TNT validates our data obtained with Kir2.1, showing that multiple approaches to Odd neural silencing have a similar effect on larval chemotaxis.

We next asked whether odor sensitivity is increased in Oddsilenced larvae as it has been shown that high odor concentrations can trigger avoidance behavior in larvae (Hallem and Carlson, 2004; Asahina et al., 2009). Thus, the phenotype observed in the che- motaxis assay could be due to Odd-silenced larvae avoiding the higher concentration of odor close to the odor source but be attracted to lower odor concentrations further away. To test this, we lowered the concentration of ACV to a 1:10 dilution. We found that lowering the concentration of ACV exacerbates the behavior of the Odd-silenced larvae, and the RI for these larvae is now significantly lower in both zones +4 and +3 compared with controls $(p<0.05$, two-way ANOVA; Fig. $4 G$ ). In fact, Odd-silenced larvae perform worse across all four zones as do the controls (Fig. 4, compare $G, D$ ). Had the Odd-silenced larvae been sensitive to the high odor concentration, we would expect the larvae to perform in a similar way as the parental strains at lower concentrations of ACV.

Next, we tested whether the Odd neurons are involved in avoidance behavior using the repellents 1-nonanol and geranyl acetate (Kreher et al., 2008). Using 1-nonanol did not result in any difference in avoidance behavior between Odd-silenced larvae and controls, suggesting that Odd neurons are not required for odor avoidance (Fig. 4H). We wondered whether this could be due to a lack of Odd neural responses to 1-nonanol. We therefore performed functional imaging using an approach similar to those described above to test odor-evoked responses to a $10^{-1}$ dilution of 1-nonanol (Fig. 4I). We found that none of the animals imaged $(N=6)$ respond to 1-nonanol. This shows that Odd neurons are not responding to 1-nonanol. We next asked whether Odd-silenced larvae behave similarly to another repellent and therefore tested larval behavior using geranyl acetate. We found a response similar to that for 1-nonanol (Fig. $4 J$ ), suggesting that silencing Odd neurons specifically impairs the behavioral response to attractive odors.

The distribution of GRASP puncta suggests that Odd neurons could respond to a broad collection of odors. Thus, we tested whether Odd-silenced larvae behave similarly to other types of odors by testing their behavioral response to another complex odor, banana mush, as well as several monomolecular odors; 2-heptanone, octanal, 1-hexanol, isoamyl acetate, and methyl acetate (Fig. 5). We first addressed whether Odd neurons respond to these odors by performing functional imaging, as described above. We find that the Odd neurons respond to all attractive odors tested (Fig. 5A,B) but, in addition to 1-nonanol, does not respond to geranyl acetate. The response profiles were similar between the odors tested (Fig. 5A), and there was no significant difference in maximum peak responses (Kruskal-Wallis test) $(N=6$; Fig. $5 B)$ among different odors except for banana mush, which elicited a slightly lower response than the other odors. We found similar chemotaxis behavior with all attractive odors tested (Fig. 5C-H).

\section{Larvae are more sensitive to odor concentration when Odd neuronal excitability is increased}

To complement the loss-of-neural function studies, we increased neural excitability in the Odd neurons using a low-activation threshold, voltage-gated sodium channel ( $\mathrm{NaChBac}$; Nitabach et al., 2006). We reasoned that if abolishing Odd neural activity impairs chemotaxis, then increasing their excitability (Oddexcited larvae) should render the larvae more efficient at navigating toward the odor source. Similar to the approach taken for silencing, we used the chemotaxis assay to address how efficiently Odd-excited larvae can reach the odor source using ACV as an attractive odor. A significantly higher proportion of Odd-excited larvae (Odd-gal4, Gal80ts UAS-NaChBac) reach the odor source in zone $+4(p<0.001$, two-way ANOVA $)$ compared with the controls (Fig. $6 A$ ).

To validate the result obtained with $\mathrm{NaChBac}$, we used an alternative method of activating the Odd neurons by expressing the warmth-activated cation channel Drosophila TRPA1 (Rosen- 

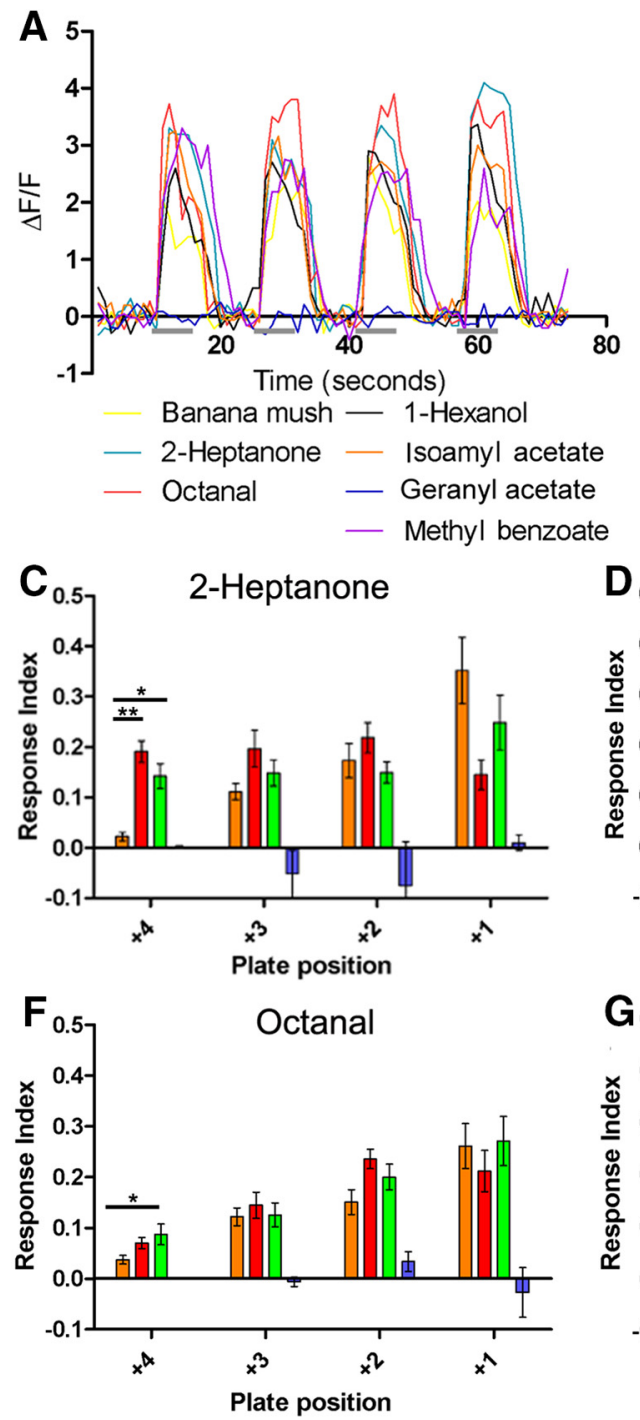

B
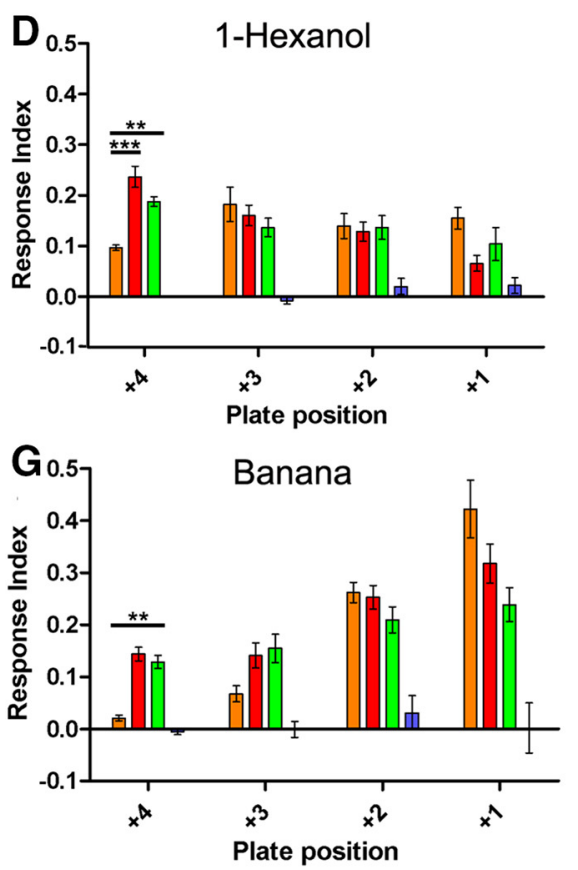
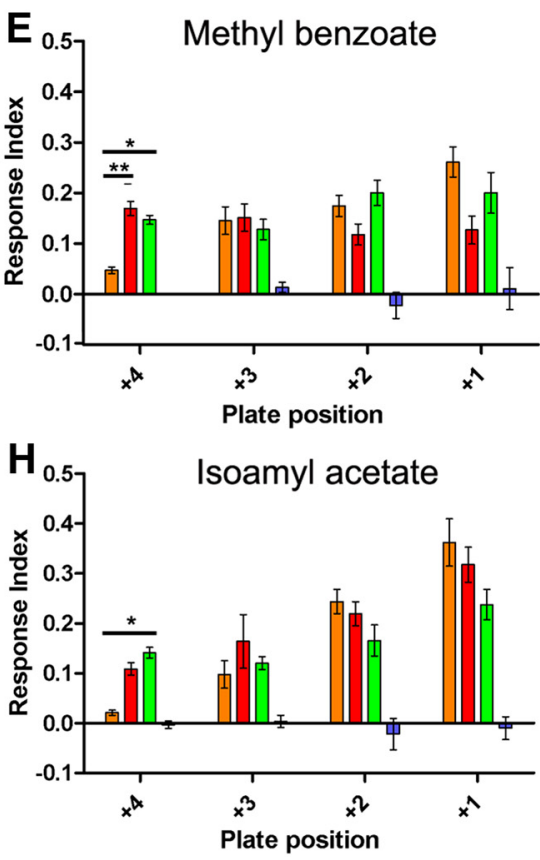

Figure 5. Behavioral and neural responses to different odors. $\boldsymbol{A}, 0$ dor-evoked responses of $0 \mathrm{dd}$ neurons expressing GCaMP6 using functional imaging to various monomolecular odors (10 ${ }^{-1}$ dilution) and banana mush. Each colored line represents the response of one Odd neuron exposed to multiple cycles of different odors. $\boldsymbol{B}$, Average maximum response of several larvae $(N=6)$ showing that all of the seven attractive odors tested generate responses in the $0 \mathrm{dd}$ neurons, whereas the two repellents 1 -nonanol and geranyl acetate both fail to elicit responses in $0 \mathrm{dd}$ neurons upon odor stimuli. $\mathbf{C}-\boldsymbol{H}$, Behavioral responses to different monomolecular odors at $10^{-1}$ dilution and banana mush. In all assays, bars represent the RI \pm SEM ( $\left.n=10\right)$. Statistical significance was calculated using a two-way ANOVA and Bonferroni post-test to calculate the degree of statistical significance. ${ }^{*} p<0.05,{ }^{* *} p<0.01,{ }^{* * *} p<0.001$. The silencing of 0 dd neurons has a similar behavioral effect for all odors tested.

zweig et al., 2005). Expressing the TRPA1 in the Odd neurons at $30^{\circ} \mathrm{C}$ resulted in a behavioral response that was similar to that of $\mathrm{NaChBac}$ (Fig. 6B). These data suggest that increasing Odd neural excitability does indeed enhance chemotaxis. We addressed whether the phenotype observed is due to an increase in larval locomotion by filming crawling larvae during the chemotaxis assay in the absence of odor. We found no difference in average speed between Odd-excited larvae and controls (Fig. 6C), showing that increasing Odd excitability does not affect the speed of locomotion. Next, we asked whether the improved performance in the chemotaxis assay is similar at lower odor concentrations. We therefore tested the behavioral response of Odd-excited larvae to a 1:10 dilution of ACV and found that enhanced chemotaxis is maintained, even at lower concentrations of odorants, compared with controls ( $p<0.001$, two-way ANOVA; Fig. $6 D$ ).

These data suggest that increasing Odd neural excitability renders the larvae more sensitive to odor concentrations. To address this further, we tested whether Odd-excited larvae are better at discriminating among different odor concentrations. We devised an assay in which the larvae can make a choice between two concentrations of odor (Fig. $6 E$ ) in a Y-shaped chamber that was $10 \mathrm{~cm}$ long and $2 \mathrm{~cm}$ wide. Larvae were positioned at the bottom of the $\mathrm{Y}$, and two different concentrations of ACV were applied to either arm of the Y. Larvae were allowed to wander for $10 \mathrm{~min}$ before the number of larvae in each arm was counted. We used our data from the chemotaxis assay, which show that larvae are more attracted to the full concentration of ACV compared with diluted ACV.

We first addressed whether the odor arena can be used for odor discrimination by testing whether larvae can navigate in this type of assay and are able to choose the arm containing the higher concentration of odor. We therefore introduced a large difference (10-fold) in odor concentration between the two arms by adding $20 \mu \mathrm{l}$ of undiluted ACV to one arm and $20 \mu \mathrm{l}$ of a $10^{-1}$ dilution of ACV to the other arm. In the chemotaxis assays, we found that both the Odd-excited larvae and the Odd-Gal4 control can chemotax and 
A

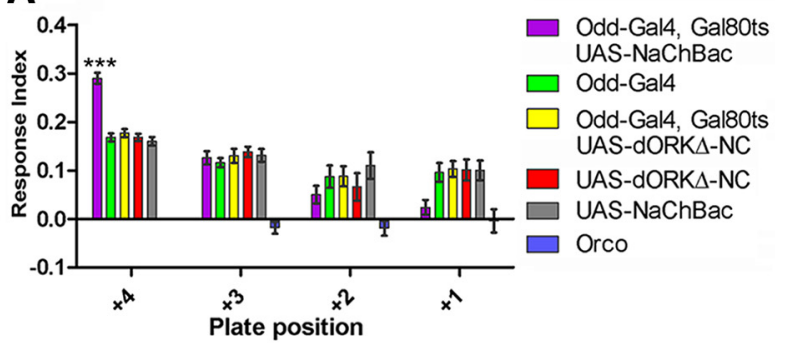

C

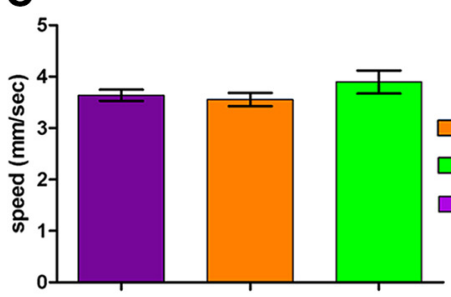

B

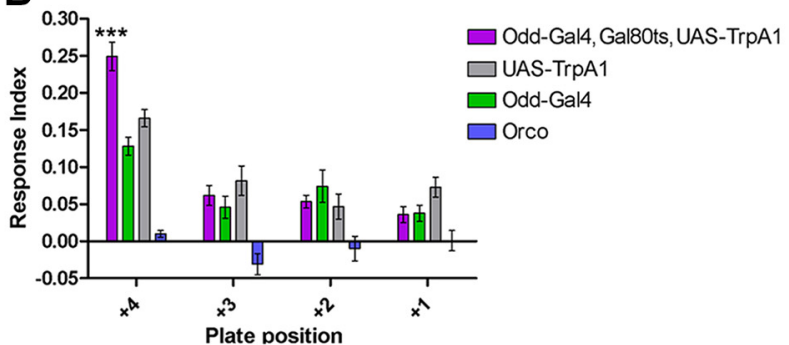

D
$\rightarrow$ Odd-Gal4, Gal80ts, UAS-NaChbac $\rightarrow$ Odd-Gal4
- Odd-Gal4, Gal80ts UAS-kir2.1
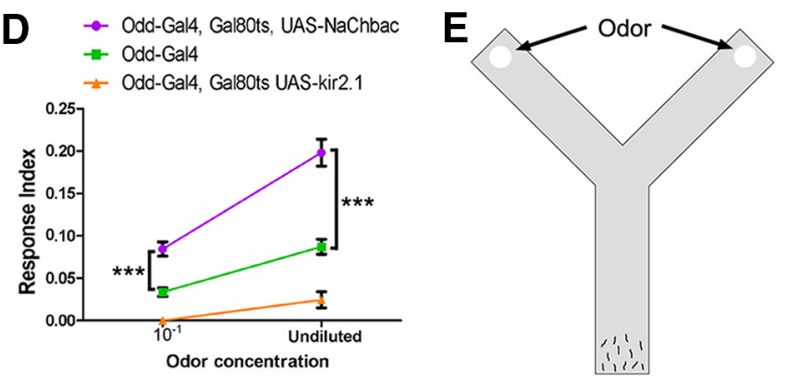

G
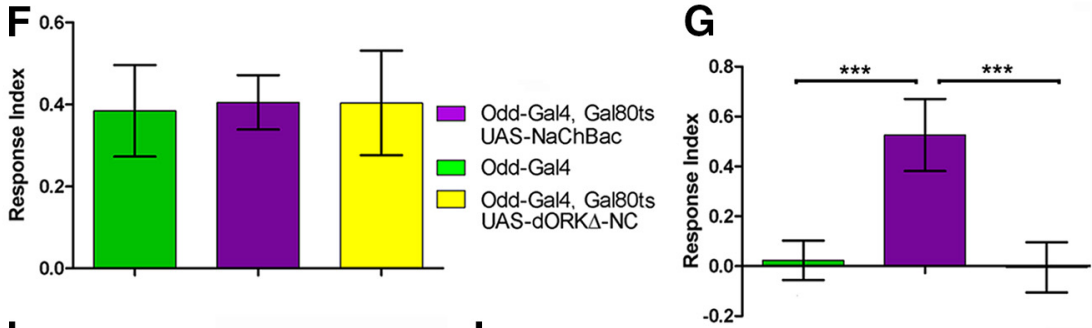

H

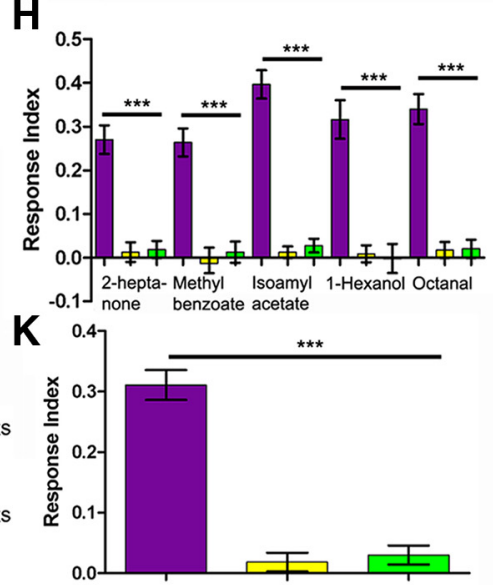

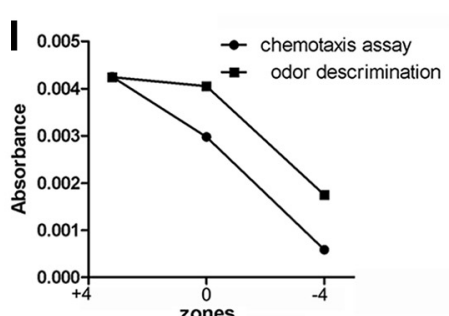

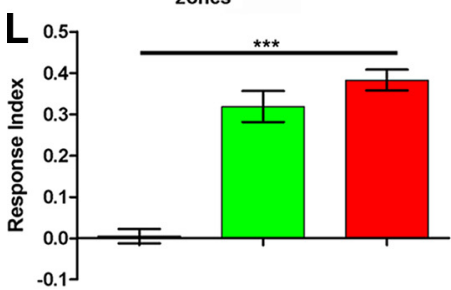

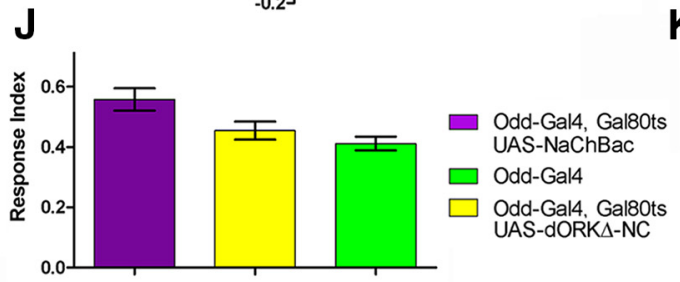

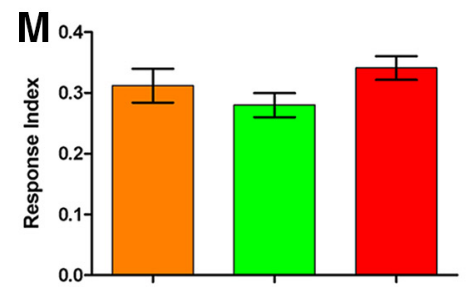

Figure 6. Odd-excited larvae show improved odor concentration discrimination. $A$, Behavioral responses to ACV of Odd-excited larvae using NaChBac (purple). The proportion of Odd-excited larvae in zone +4 is significantly larger compared with controls. $\boldsymbol{B}$, Behavioral responses to ACV using $\operatorname{TrpA1}$ to excite the $0 \mathrm{dd}$ neurons. Odd-excited larvae are significantly better $(p<0.001)$ at locating the odor source in zone +4 compared with controls. The RI response is calculated for each zone of the plate ( $x$-axis). Each bar represents the RI \pm SEM $(n=10)$. A two-way ANOVA was used to calculate statistical significance and Bonferroni post-test to calculate the degree of statistical significance ${ }^{* *} p<0.001$. C, Locomotion speed of larvae in the absence of odor calculated from the speed tracks generated by MWT. Each bar represents average locomotion over a 4 min period ( $y$-axis) \pm SEM $\left(N=7\right.$ ) (Kruskal-Wallis test combined with a Bonferroni post-test). $D$, Comparison between the behavioral responses at full concentration and $10^{-1}$ dilution of ACV. Odd-excited larvae still perform better at lower odor concentrations. Kruskal-Wallistest combined with a Bonferroni post-test, $p<0.001 . E$, Schematic representation of the odor discrimination assay. $\boldsymbol{F}$, Behavioral responses to a 10-fold difference in odor concentration, showing that larvae can locate the arm containing the higher concentration of odor. $\boldsymbol{G}$, Behavioral responses to a fourfold difference in odor concentration. The Odd-excited larvae (purple) are statistically better $\left.{ }^{* * *} p<0.001\right)$ at choosing the higher odor concentration compared with controls. Each bar represents the RI for the higher odor concentration \pm SEM $(N=10)$. Statistical significance was calculated using a one-way ANOVA and Bonferroni post-test. $\boldsymbol{H}$, Behavioral responses to a fourfold difference in odor concentration of a selection of the monomolecular odorants used in the chemotaxis assay. Regardless of the odor tested, Odd-excited larvae can correctly locate the arm with the higher odor concentration, whereas control larvae cannot differentiate between the fourfold differences in odor concentration between the two arms. Statistical significance was calculated using a one-way ANOVA and Bonferroni post-test. II, Odor concentration discrimination was also tested using an arena similar to that for the chemotaxis assay butreplacing the control odor in zone -4 with a fourfold diluted ACV solution. Using Fourier transform infrared spectroscopy, we show that an odor gradient is generated within this arena. However, the addition of diluted odor to the control side increases the overall concentration of odor within the arena and decreases the slope of the gradient between zone +4 and zone 0 compared with the chemotaxis assay. J, $K$, Using the $0 \mathrm{dd}$-excited larvae as a control, we show that we obtain a similar behavioral response in this type of odor arena compared with the $Y$ maze.J, Similar to the Y maze, Odd-excited larvae and control larvae can locate the odor source in the chemotaxis arena when a 10-fold difference in odor concentration is applied to either side of the arena. $\boldsymbol{K}$, A fourfold difference in odor concentration rendered the control larvae incapable of discriminating between the two odor concentrations, whereas $0 \mathrm{dd}$-excited larvae maintained their ability to choose the side containing the higher odor concentration. $L$, Odd-silenced larvae cannot differentiate between a sixfold concentration difference in ACV, whereas both control larvae choose the side with the higher concentration of ACV.M, All three genotypes can locate the source of the higher odor concentration when larvae are presented with a 10 -fold difference in the concentration of ACV. Statistical significance was calculated using a one-way ANOVA and Bonferroni post-test ${ }^{* *} p<0.001$. 


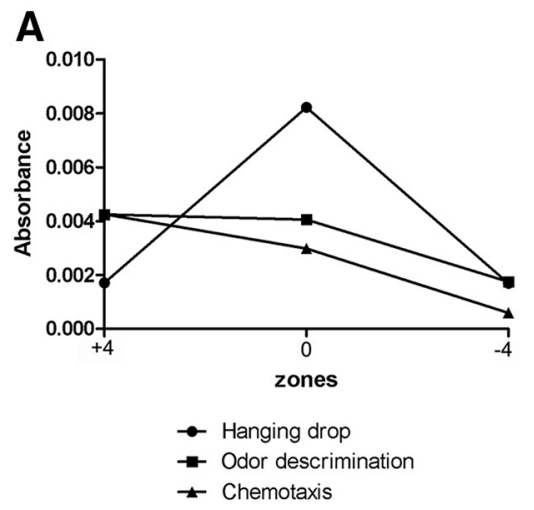

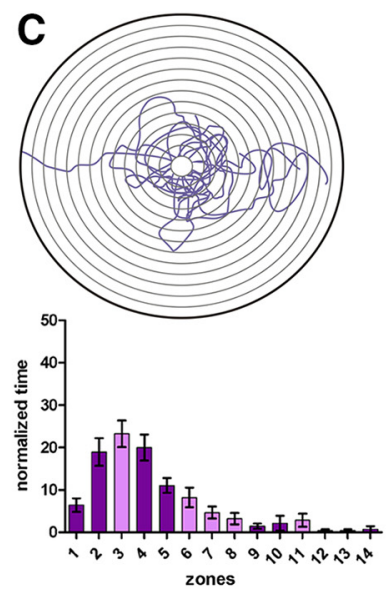

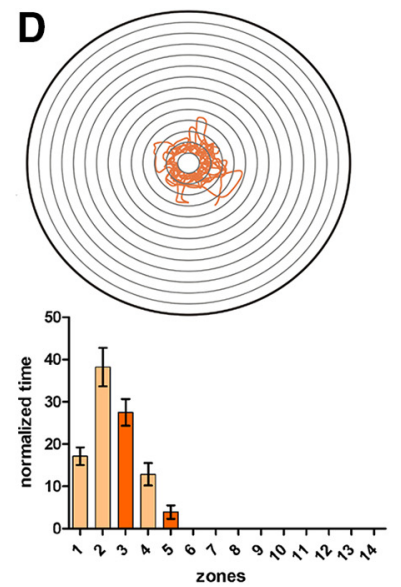

Figure 7. Odd neural modulation does not impair navigation at high odor concentrations. $\boldsymbol{A}$, Fourier transform infrared spectroscopic measurements of the odor concentration distribution in the gas phase within the hanging drop arena compared with the chemotaxis and odor discrimination arena. Concentration is high near the odor source in the middle of the plate compared with measurements taken at positions similar to those in zones +4 and -4 . $\boldsymbol{B}-\boldsymbol{D}$, Behavioral responses in a hanging drop assay $(n=15)$. Top, Locomotion tracks. Bottom, Bar plots representing the mean ( \pm SEM) occupancy in respect to the odor source. Bars of lighter shade represent statistically significant occupancy compared with the control. $\boldsymbol{B}, 0 \mathrm{dd}-\mathrm{Gal} 4$. $\boldsymbol{C}, 0 \mathrm{dd}$-excited. $\boldsymbol{D}, 0 \mathrm{dd}$-silenced larvae. A Wilcoxon signed rank test corrected for multiple comparisons was used to calculate statistical significance.

locate the odor source when ACV is diluted 1:10 (Fig. 6D). Thus, we know that larvae can respond to lower concentrations of ACV. Under these conditions, both the Odd-excited larvae and the controls consistently chose the arm containing the higher odor concentration (NS, two-way ANOVA; Fig. $6 F$ ). This clearly demonstrates that larvae can navigate to find the source of higher odor concentration within this type of assay. However, when the odor concentration was lowered to a fourfold difference the controls were no longer able to differentiate between the two arms, and the distribution of control larvae were similar between the two sides (RI =0; Fig. $4 G)$. The Odd-excited larvae, on the other hand, consistently chose the arm with the higher concentration ( $p<0.001$, two-way ANOVA). This shows that Odd-excited larvae are better at odor concentration discrimination, and this suggests that the Odd-excited larvae are more sensitive to relative differences in odor concentration. Can Oddexcited larvae discriminate between a fourfold differences in odor concentration for a range of odors? To address this, we chose the same panel of odors as those shown to elicit a neural response (Fig. 5). We find that Odd-excited larvae can indeed discriminate between a fourfold difference in odor concentrations of all odors tested, whereas the distribution of control larvae is similar between the two arms (Fig. 6H).

If Odd-excited larvae are better at odor discrimination, it stands to reason that Odd-silenced larvae should perform worse at this task compared with controls. We initially attempted to address this using the Y maze but found that few Odd-silenced larvae navigated into the two arms. Therefore, we chose an alternative odor discrimination assay based on the circular arena used for the chemotaxis assay. In fact, we maintained all the same parameters as for the chemotaxis arena, except, instead of adding a control solution to the Whatmann paper in zone -4 , we added the diluted concentration of ACV. First, we confirmed that the odor concentrations at zones $+4,0$, and -4 are different by measuring the odor concentrations in gas phase at zones $+4,0$, and -4 using FTIR (Fig. $5 I$ ). We found that the odor concentration is indeed different between the two outer zones, but, as expected, the slope of the gradient between zone +4 and zone -4 is shallower due to the addition of odorant in zone -4 . As a control, we first tested Odd-excited larvae in this type of assay and counted the number of larvae entering zones 2, 3, and 4. We calculated an RI by deducting the total number of larvae in zones $-4,-3$, and -2 from the total number of larvae in zones $+4,+3$, and +2 , and divided this number with the total number of larvae in the assay (50). Gratifyingly, we obtained a result similar to that observed in the Y maze (Fig. $6 J, K$ ), in that Odd-excited larvae can differentiate between a fourfold difference in odor concentration whereas control larvae cannot. Similar to the Y maze, all three genotypes can differentiate between a 10-fold odor concentration difference (Fig. 6K). The use of the alternative chemotaxis arena further validates our results obtained for the Odd-excited larvae. Next, we addressed whether Odd-silenced larvae can discriminate among a range of odor concentration differences and found that Odd-silenced larvae cannot discriminate between odor concentration differences up to sixfold, whereas control larvae can (Fig. 6L). Thus, Odd-silenced larvae are worse at odor concentration discrimination compared with controls. All three genotypes can differentiate between a 10-fold odor concentration difference (Fig. 6M), demonstrating that Oddsilenced larvae can choose the side containing the higher odor concentration, given a sufficient difference in concentration.

\section{Modulating Odd neural function does not affect chemotaxis at high odor concentrations}

Our behavior experiments show that modulating Odd neural activity influences chemotaxis. However, it does not address how Odd neural activity affects the ability of the larvae to stay near an odor source where odor concentrations are high. To address this, we used a hanging drop assay (Asahina et al., 2009) in which the larvae are placed directly under a drop of odor suspended from the lid of the chamber. The key difference between this assay and the chemotaxis assay is that in the hanging drop assay the larvae cannot reach the odor source and therefore continue to search for the source of the odor. In the chemotaxis assay, the larvae stay on the odor source once they have reached it. Thus, the chemotaxis assay is not suitable to address chemotaxis behavior at high odor concentrations since the larvae would stop navigating as soon as they reach the odor source. In addition, we found that the concentration of odorant is much higher near the odor source in the hanging drop assay than near the odor source in the chemotaxis assay (Fig. 7A), despite the fact that a similar amount of concentrated ACV is added in both assays. Thus, the hanging drop assay is ideal for testing navigation at high odor concentrations.

In the hanging drop assay, wild-type larvae have been shown to continuously circle the odor drop within a narrow range (Asa- 
hina et al., 2009). We adopted the approach of Asahina et al. (2009) and divided a $9 \mathrm{~cm}$ plastic dish into $0.25 \mathrm{~cm}$ concentric circles from the odor drop, and measured how much time the larvae spend in each circle. We also mapped the locomotor tracks. We used the Odd-Gal4 as a control since this line is common to both the Odd gain-of-neural function and loss-of-neural function genotypes. Odd-Gal4 larvae behave similarly to wild-type larvae (Asahina et al., 2009; Fig. 7B), in that they circle the hanging drop and primarily stay within a $1.5 \mathrm{~cm}$ radius of the odor drop. Odd-excited larvae are also able to navigate within the vicinity of the hanging drop (Fig. 7C) and, in terms of the time spent in the closest two zones, are not statistically different from controls. Thus, they are not repelled by high odor concentrations. However, Odd-excited larvae also navigate away from the odor drop. Therefore, they spend significantly more time in the zones further away from the odor drop compared with the controls $[p=0.0235$ (zone 6), $p=0.0145$ (zone 7$), p=0.083$ (zone 8$), p=$ 0.0429 (zone 11), Wilcoxon test]. This could suggest that Oddexcited larvae are not as sensitive to a decrease in odor concentration, probably due to stimulus saturation. If this is the case, then we would expect that Odd neural silencing would have the opposite effect. Indeed, when Odd-silenced larvae are tested in the hanging drop assay (Fig. $7 D$ ), they spend significantly more time underneath the odor drop and in zone 1 and $2(p=0.0043$ for zone $1 ; p=0.0028$ for zone 2) compared with the controls. This confirms our previous finding (Fig. $4 G$ ), which shows that Odd-silenced larvae are not repelled by high concentrations of odor. Furthermore, these data clearly demonstrate that modulating Odd neural function does not prevent navigation near the odor source, where odor concentrations are high.

\section{Manipulating Odd neural activity affect behavior during chemotaxis}

Our data suggest that chemotaxis behavior may be altered when Odd neural function is manipulated. To investigate this further, we filmed larval behavior in the chemotaxis assay. We focused exclusively on the area between zones 0 and +4 (Fig. $8 A$ ). We analyzed our movies using FIMTrack (Risse et al., 2013) to quantify the number of stops, lateral head swings, and turns performed by the larvae during chemotaxis. First, we compared the frequency of head swings per minute between Odd-silenced larvae and controls. It has previously been found (GomezMarin et al., 2011) that head swings toward a high odor concentration is rarely followed by a second head swing whereas a first head swing toward low odor concentration is frequently followed immediately by a head swing in the opposite direction. Thus, the frequency and the distribution of left/right head swings are indicative of the ability of the larvae to measure the odor concentration between two points in space. We therefore separate single head swings and double head swings in the analysis.

Odd-silenced larvae execute both single and double head swings (gray boxes) more frequently than control larvae (Fig. $\left.8 B-B^{\prime \prime}\right)$. Odd-silenced larvae carry out more than three times as many double head swings per minute compared with the controls $(p<0.001$, one-way ANOVA; Fig. $8 C$ ). In the absence of odor, the rate of double head swings is similar between Odd-silenced larvae and controls (Fig. $8 \mathrm{C}$ ), showing that the increase in head swings is odor dependent. The frequency of double head swings goes up in the absence of odor both for the Odd-silenced larvae and the controls (Fig. 8C). There is also a change in frequency of single head swings between Odd-silenced larvae and the controls in the presence of odor $(p<0.05$; Fig. $8 D)$. These data suggest that silencing the Odd neurons impairs odor sampling broadly, since the frequencies of both single and double head swings are affected. Importantly, this behavior phenotype is odor dependent, showing that silencing the Odd neurons does not modify head swing frequency permanently.

Lateral head swings are preceded by a stop in forward motion (Gomez-Marin et al., 2011), and we would therefore expect that Odd-silenced larvae would show a similar increase in the frequency of stops per minute. This is indeed the case, and we find a distribution of stops per minute for the three genotypes tested that is similar to that observed for lateral head swings $p<0.001$, one-way ANOVA; (Fig. 8E).

Lateral head swings precede turning (Gomez-Marin et al., 2011), and if the Odd-silenced larvae carry out more head swings, it would stand to reason that they turn more frequently. We therefore used FIMTrack to correlate stops and lateral head swing with an instantaneous change in orientation to address whether the increased frequency in lateral head swings observed for the Odd-silenced larvae results in an increase in directional turning. Again, we find that the Odd-silenced larvae carry out more turns per minute than the controls $p<0.001$, one-way ANOVA; (Fig. $8 F$ ), whereas turning frequency is similar among the three genotypes in the absence of odor. FIMTract also maps the entire sequence of movements of a given larvae over time. We used these to compare tracks between Odd-silenced larvae and controls $(N=10$; Fig. $8 G-I)$. As expected, the increase in turn frequency for the Odd-silenced larvae results in locomotion tracks that are more convoluted compared with the relatively straight tracks of the controls. The Odd-silenced larvae travel in circles as opposed to a more straight line toward the odor source.

Collectively, these data could suggest that the Odd-silenced larvae are not able to measure the difference in odor concentration during head swings, causing an increase in odor sampling frequency and a failure to navigate efficiently toward a source of odor.

If this is indeed the case, we would expect that larvae in which Odd neural excitation is increased to be more proficient at odor tracking. To address this, we analyzed the chemotaxis behavior of the Odd-excited larvae in a similar manner to the Odd-silenced larvae. When we plotted head swings against time, it is clear that the Oddexcited larvae carry out fewer double and single head swings during chemotaxis compared with their behavior in the absence of odor (Fig. $9 A, A^{\prime}$ ). In the presence of odor, the number of double head swings is significantly lower than that in the controls $(p<0.05$, one-way ANOVA; Fig. 9B), and, similar to the Odd-silenced larvae, this is an odor-specific response since the number of double head swings does not differ among the different genotypes in the absence of odor (Fig. 9B). A similar situation is observed with single head swings between Odd-excited larvae and controls $(p<0.05$, one-way ANOVA; Fig. 9C). The single-head swing phenotype is also odor dependent (Fig. 9C). The frequency of stops per minute is also lower for Odd-excited larvae compared with controls $p<0.05$, one-way ANOVA (Fig. 9D). Interestingly, the frequency of turns for Odd-excited larvae is not significantly lower than that of the controls in the presence of odor (Fig. 9E). Thus, Odd-excited larvae do turn as frequently as controls but do not stop and carry out lateral head swings before turning as frequently as the controls. The locomotion tracts are therefore similar to that seen for the Odd-Gal4 control (compare Figs. $8 \mathrm{H}, 9 \mathrm{~F}$ ). These data would suggest that Odd-excited larvae do not need to use head swings as often as controls to measure the odor gradient and instead extract sufficient odor information during runs. 
A
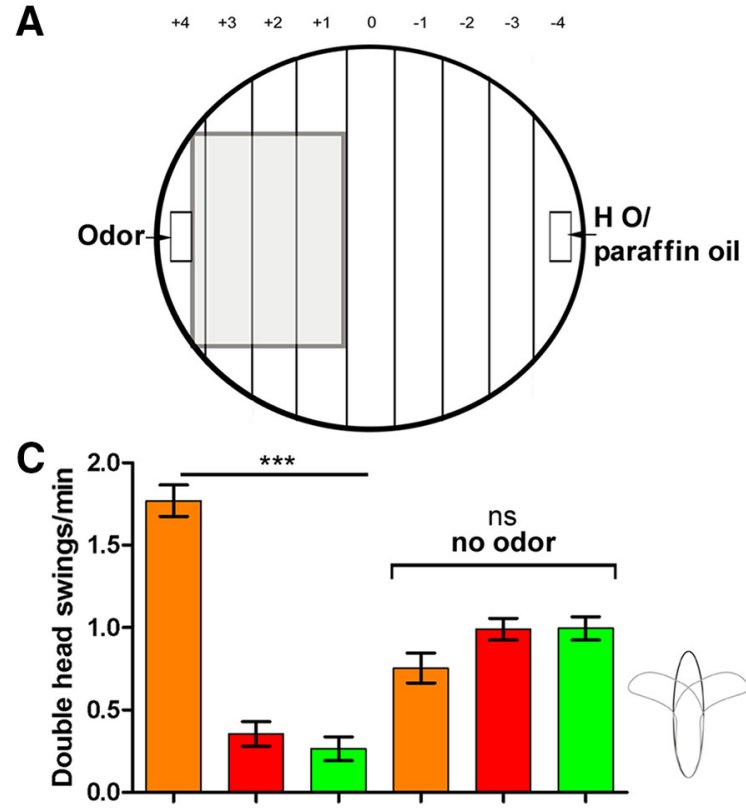

D

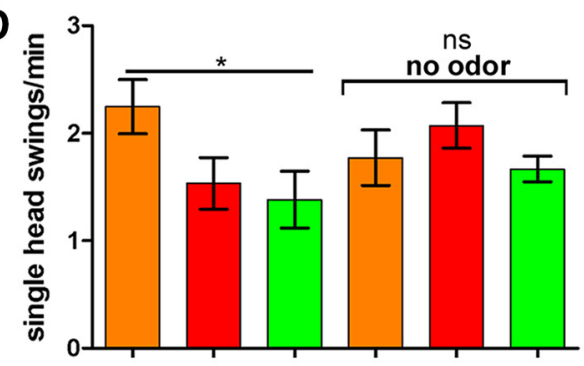

$\mathbf{F}$

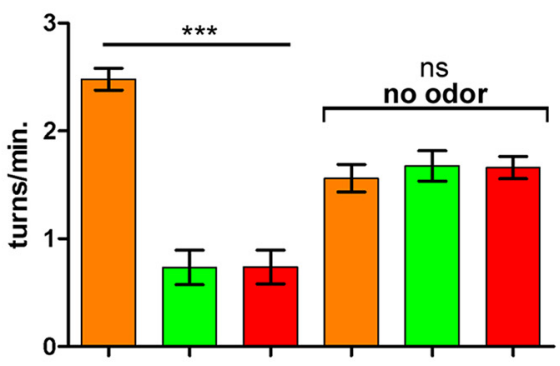

Odd-Gal4, Gal80ts, UAS-kir2.1 $\square$ Gal80ts, UAS-kir2.1 Odd-Gal4
B

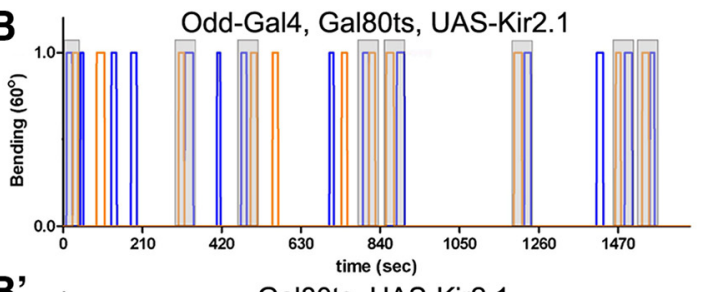

B' Gal80ts, UAS-Kir2.1
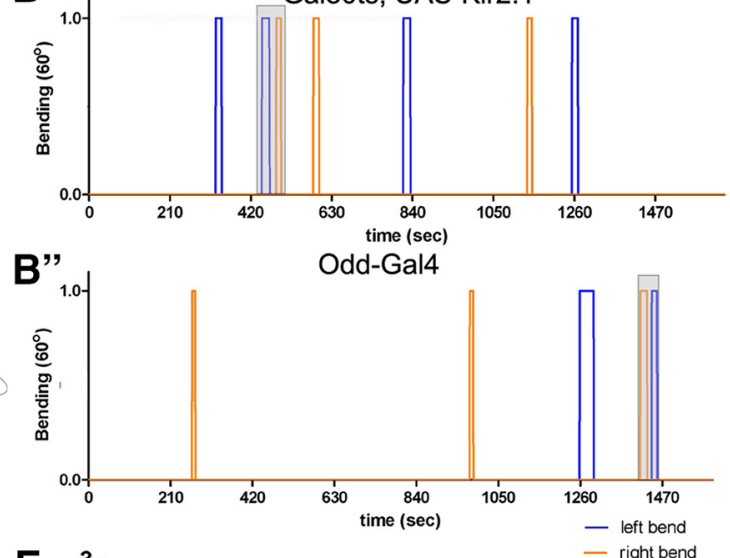

E
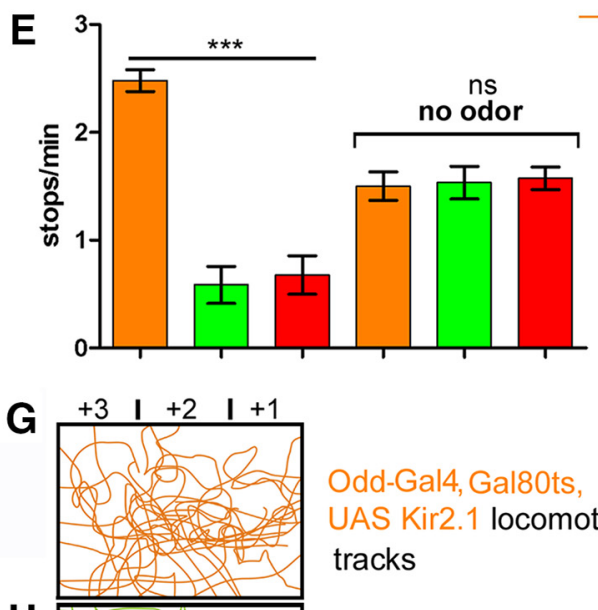

Odd-Gal4, Gal80ts, UAS Kir2.1 locomotion tracks

H

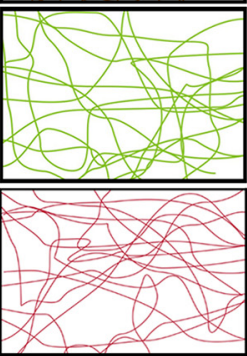

Odd-Gal4 locomotion tracks

Odd-Gal4, Gal80ts

locomotion tracks

Figure 8. Odd-silenced larvae show increased frequency of lateral head swings and turning during chemotaxis. $A$, Schematic representation of the area filmed (gray box) during chemotaxis. $\boldsymbol{B}-\boldsymbol{B}^{\prime \prime}$, Distribution of head swings of a single larva. FIMtrack values were plotted against time using 4 min film segments. Gray boxes labels double head swings. $\boldsymbol{B}^{\prime}, \boldsymbol{B}^{\prime \prime}$, Odd-silenced larvae perform more single and double head swings compared with the Gal80ts, UAS-Kir2.1 control ( $\left.\boldsymbol{B}^{\prime}\right)$, and the 0dd-gal4 control ( $\left.\boldsymbol{B}^{\prime \prime}\right)$. $\boldsymbol{C}$, Quantitative measurement of double head swings. Bar represents double head swings/min \pm SEM $(N=15)$. The number of double head swings/min is significantly higher for Odd-silenced larvae (orange) compared with both parental line controls $(p<0.001)$. In the absence of odor, there is no difference between $0 \mathrm{dd}$-silenced larvae and controls. $D$, Number of single head swings performed by the Odd-silences larvae in the presence and absence of odor. The number of single head swings is statistically higher in $0 \mathrm{dd}$-silenced larvae compared with controls $(p<0.05)$ in the presence of odor but is not statistically significant compared with the controls in the absence of odor. $\boldsymbol{E}$, Number of stops in larval locomotion in the presence and absence of odor $(p<0.001)$. Bar represents the number of stops per minute \pm SEM $(N=15)$. Odd-silenced larvae stop significantly more frequently than both control lines in the presence of odor $(p<0.001)$. whereas the number of stops per minute is similar among all three genotypes in the absence of odor. $\boldsymbol{F}$, Average number of turns of larvae in the presence and absence of odor. $0 \mathrm{dd}$-silenced larvae turn more frequently compared with controls $(p<0.001)$. Bar represents the number of turns per minute $\pm S E M(N=15)$. A Kruskal-Wallis test was used for all statistical calculations. $\mathbf{G}-\mathbf{I}$, Locomotion tracks from multiple larvae $(N=10)$ generated from FIMtrack. 

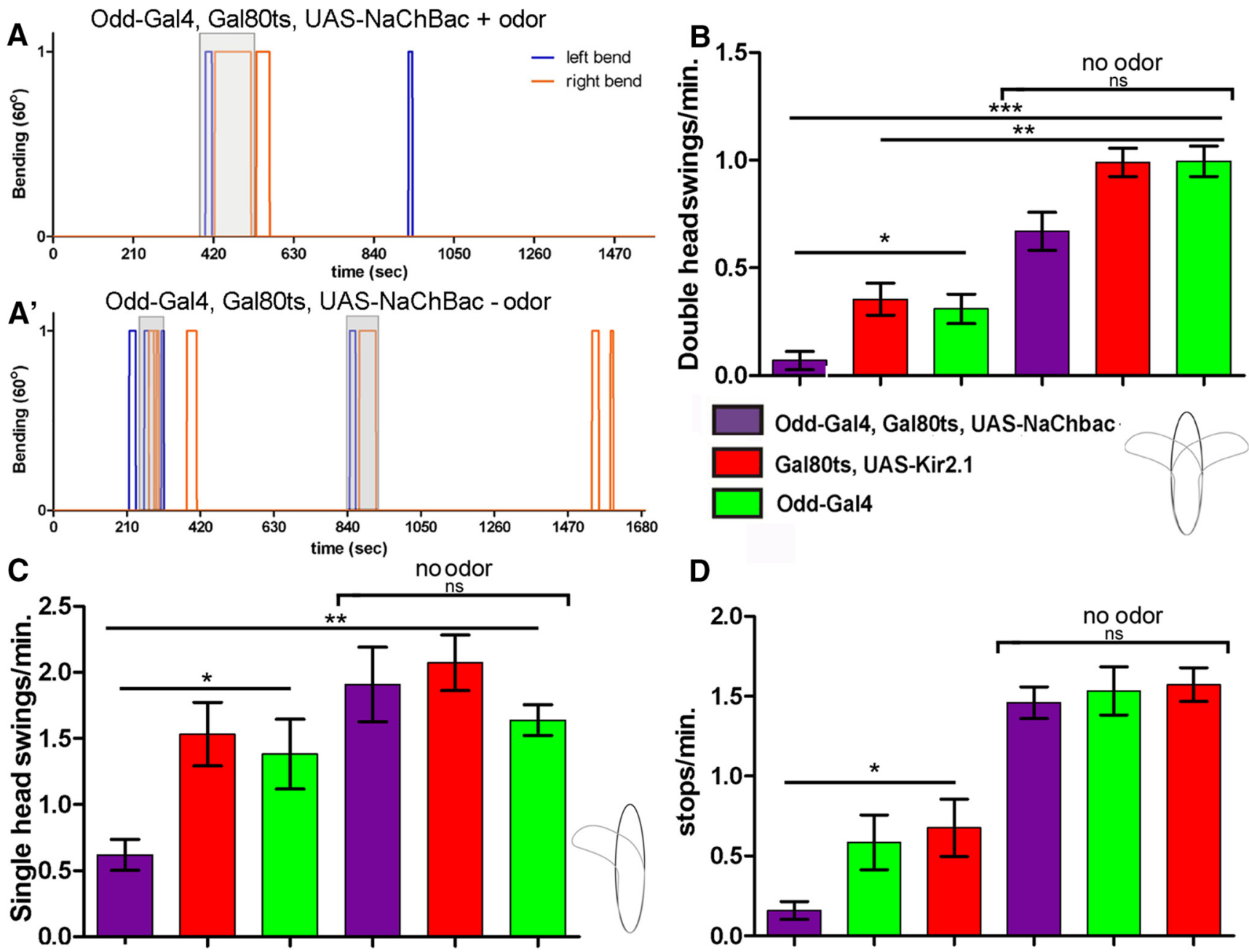

D
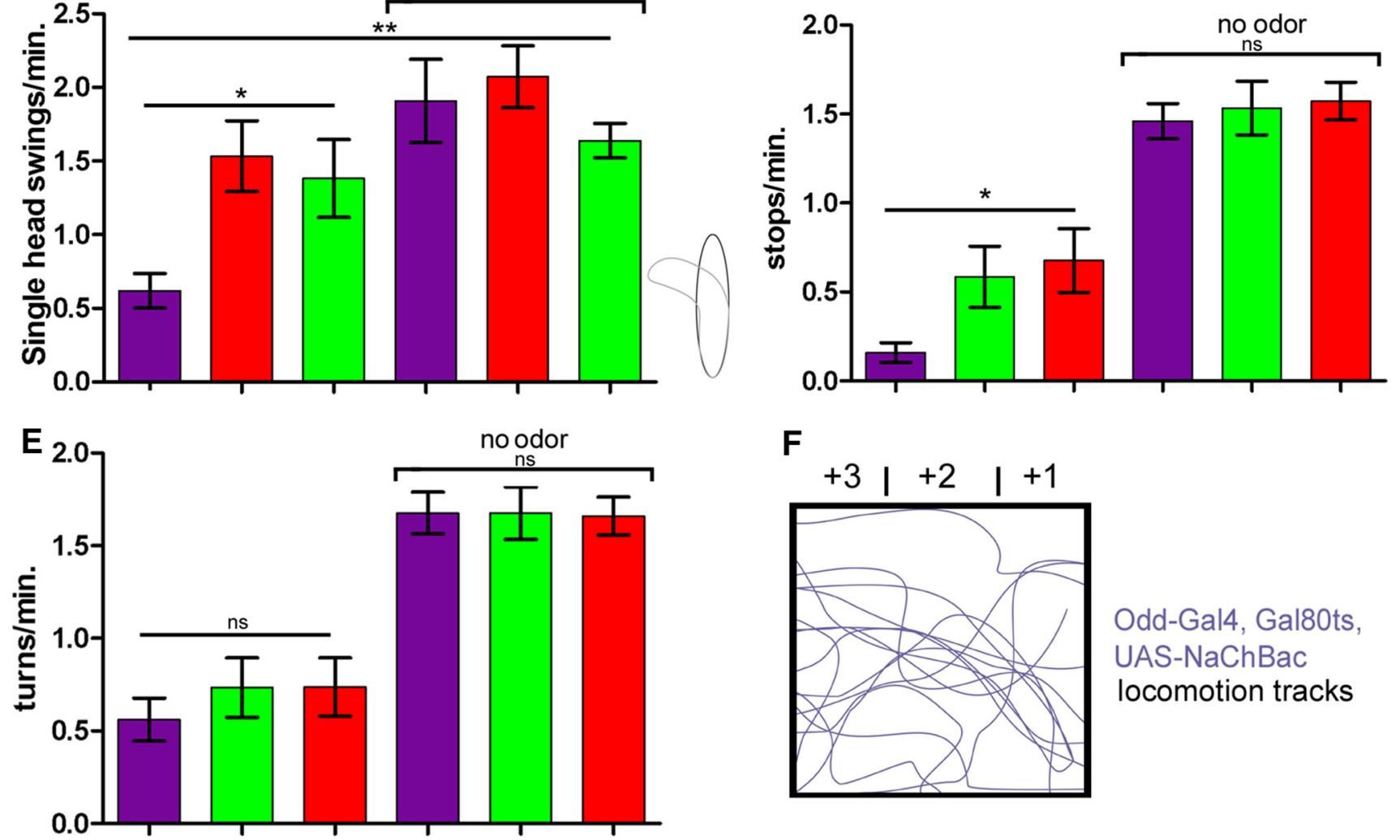

Figure 9. Odd-excited larvae perform fewer head swings during chemotaxis. $\boldsymbol{A}, \boldsymbol{A}^{\prime}$, Number of head swings for the $0 \mathrm{dd}$-excited larvae in the presence of odor $(\boldsymbol{A})$ and in the absence of odor $\left(\boldsymbol{A}^{\prime}\right)$ of single larvae. FIMtrack values were plotted against time using 4 min film segments. Gray boxes label double head swings. $B$, Quantitative measurement of number of double head sweeps between Odd-excited larvae and controls in the presence and absence of odor. Odd-excited larvae perform statistically fewer double head swings $(p<0.5)$ compared with controls in the presence of odor, whereas all three genotypes carry out similar numbers of double head swings in the absence of odor. Odd-excited larvae also carry out significantly fewer head swings compared to controls in the absence of odor $(p<0.001)$. In addition the controls perform fewer head swings in the presence of odor compared to the number of head swings in the absence of odor ( $p<0.01)$. Each bar represents the number of double head swings per minute \pm SEM $(N=15)$. C, Number of single head swings of Odd-excited larvae compared with $0 \mathrm{dd}$-silenced larvae and controls in the presence and absence of odor. There is a statistical difference between $0 \mathrm{dd}$-excited larvae and controls $(p<0.05)$ in the presence of odor, as well as significant difference between $0 \mathrm{dd}$-excited larvae in the presence of odor, and between 0dd-excited larvae and controls in the absence of odor $(p<0.01)$. The number of single head swings between $0 \mathrm{dd}$-excited larvae and controls in the absence of odor is not significantly different. Each bar represents the number of single head swings per minute \pm SEM $(N=15)$. $D$, Average number of stops in forward locomotion per minute. Bar represents the number of stops per minute \pm SEM $(N=15)$. Odd-excited larvae stop significantly fewer times compared with control larvae $(p<0.05)$ in the presence of odor but not in the absence of odor. $\boldsymbol{E}$, Average number of turns of larvae in the presence and absence of odor. Bar represents the number of turns per minute \pm SEM $(N=15)$. The average turn rate is similar between $0 \mathrm{dd}$-excited larvae and controls both in the presence and absence of odor. $\boldsymbol{F}, 0$ dd-excited larval locomotion tracks from multiple larvae $(N=10)$. Kruskal-Wallis test was used for all statistical calculations. 


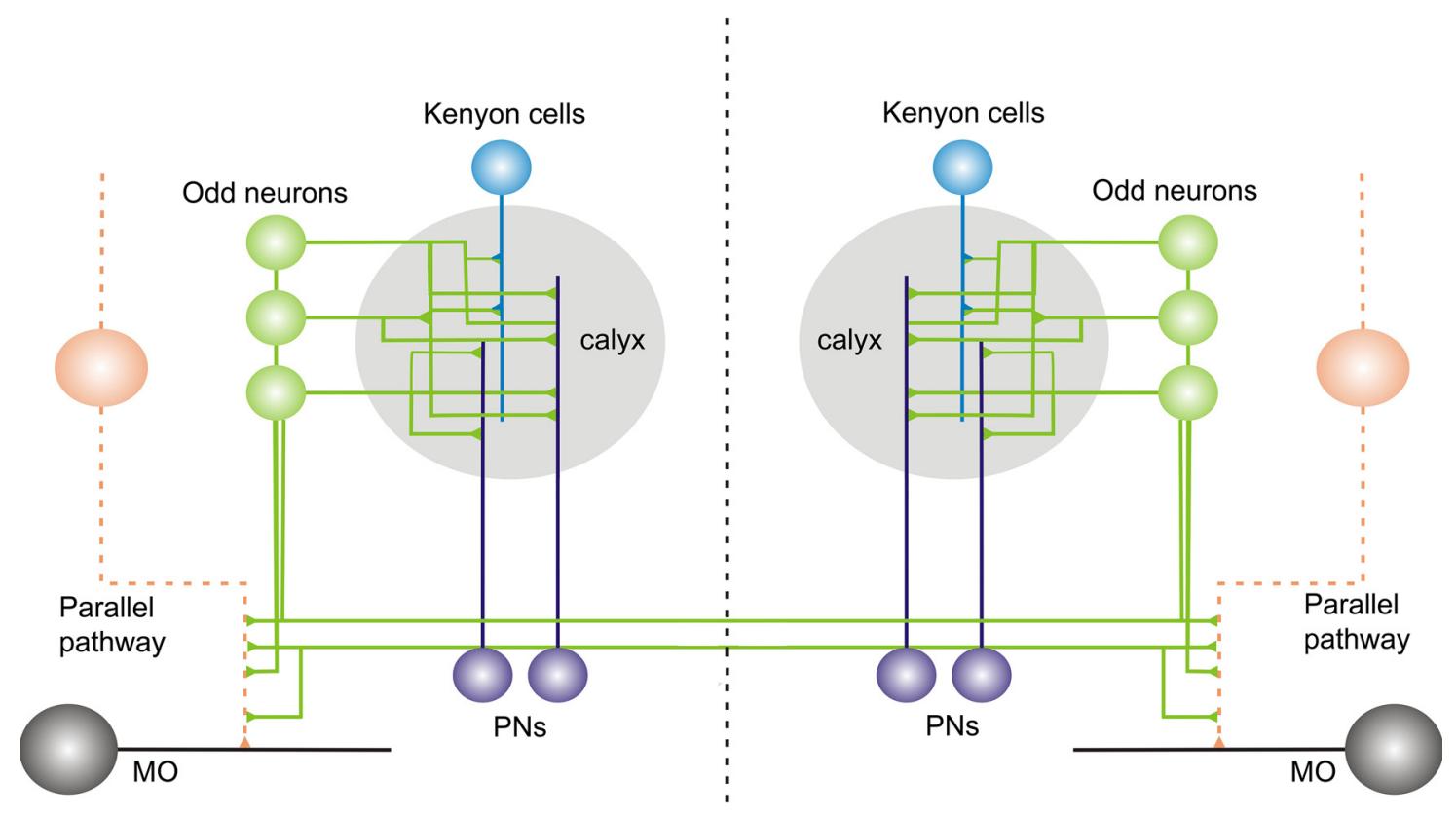

Figure 10. Chemotaxis circuit model. Odd neurons (green) receive input from PNs (purple) and Kenyon cells (light blue) at the level of the calyx. In turn, $0 \mathrm{dd}$ neurons synapse onto and provide background excitation to a parallel circuit (orange) required for chemotaxis. These neurons could either directly or indirectly synapse onto motor output (black) neurons.

\section{Discussion \\ Odd neurons form part of an olfactory circuit downstream of the mushroom body}

While significant progress has been made in understanding how odors are encoded within the first stages of olfactory processing, less is known about the circuitry that translates this information into behavioral output. Here we provide evidence that neurons of the Odd lineage form part of an olfactory circuit downstream of the PNs in the mushroom body.

Detailed morphological analysis of single Odd neurons shows that three of the Odd neurons extend dendrites into the calyx, suggesting a role in odor processing. Using in vivo functional imaging of the Odd neurons, we show that a subset of these cells does indeed respond to a broad range of attractive odors but not to odors that act as repellents. Using genetically encoded dendritic markers and GRASP labeling, we demonstrate that the odor-evoked responses of Odd neurons may be driven via direct connections to PNs. The distribution of GRASP puncta is fairly uniform across the calyx, and each Odd neuron forms extensive arbors in the calyx, suggesting that the Odd neurons probably receive input from a number of PNs. We also demonstrate reconstitution of GFP between Kenyon cells and the Odd neurons. Presynaptic densities have been observed in the Kenyon cell dendrites (Christiansen et al., 2011), and, while the extent of labeling between Kenyon cells and the Odd neurons is less extensive than that seen between the Odd neurons and PNs, it suggests that the Odd neurons are connected with Kenyon cells. In that case, these connections must be dendro-dendritic synapses, and, as such, it is not possible to infer directionality from the GRASP studies. Gomez-Marin et al. (2011) argue that there should be a shortterm memory component to chemotaxis as the larvae associates body position with odor stimuli intensity during head swings. It has also been shown that larvae can form odor intensity-specific memories (Mishra et al., 2013). Whether Odd neurons are required for amplifying Kenyon cell responses to input from the PNs or Kenyon cells modulate Odd neurons is not known. However, the connectivity between the Odd neurons and the Kenyon cells could provide a substrate for integrating memory with odor stimuli intensity.

\section{Odd neurons increase larval sensitivity to odor}

Our chemotaxis behavior analysis shows that silencing Odd neurons impairs the competence of the larvae to locate an attractive odor, and this is associated with an increase in frequency of head swings and more frequent turns. Consequently, larvae navigation is more convoluted compared with that of controls. Conversely, increasing Odd neural excitability improves larval chemotaxis due to better odor concentration discrimination. The frequency of head swings drops as they can navigate more effectively. There is a similar effect on both double and single head swings for both genotypes, suggesting that modulating Odd neural function impairs odor sampling broadly and is observed specifically in response to odor. This is the first example of an MB-associated higher-order pathway that controls behavior during chemotaxis in the larvae. Since larval aversive behavior is normal and Odd neurons do not respond to repellent odors, our data provide compelling evidence that Odd neurons are not required for aversive behavior.

Several of our experiments suggest that manipulating the Odd neurons alters the sensitivity of the olfactory system to low concentrations of odor. First, the increase in sampling frequency is evident from the onset of larval chemotaxis further from the odor source where odor concentrations are comparatively lower than near the odor source. The increase in head swings is therefore a behavior manifested at lower odor concentrations. Furthermore, the frequency of head swings increases for Odd-silenced larvae and controls in the absence of odor. This shows that larvae execute more head swings when olfactory inputs are missing, presumably in an attempt to extract information about the odor environment. This suggests that increasing the frequency of odor sampling is a general strategy adopted by larvae when odor stimuli are low or missing. The phenotype of the Odd-silenced larvae exhibited in the presence of odor is stronger than that observed in the absence of odor. This would suggest that the detection of odor 
but the inability to extract sufficient information from the odor space further promotes odor sampling.

Second, increasing the excitability of Odd neurons enhances odor concentration discrimination, and the larvae do not need to sample the odor space as frequently as control larvae (Fig. 6). This is presumably because increased sensitivity to differences in odor concentrations enables the larvae to extract sufficient information about the odor space during runs. Interestingly, stereo olfaction improves the accuracy of larval chemotaxis (Louis et al., 2008b), and it is possible that the ability of the Odd-excited larvae to discriminate among odor concentrations improves stereo olfaction, allowing them to turn toward the odor source during runs.

Finally, the behavior of the larvae in the hanging drop assay clearly demonstrates that high odor concentrations do not affect navigation near the odor source in Odd-silenced and excited larvae since both genotypes can navigate near the odor drop (Fig. 7). However, Odd-silenced larvae remain closer to the hanging drop compared with controls, suggesting that sensory input is sufficiently lowered to allow for a better response to changes in odor concentrations. Larvae in which the Odd neural excitability is increased navigate further from the odor source, presumably due to stimulus saturation. The fact that these larvae can navigate near the odor source suggests that stimulus saturation is not sufficiently high to promote repulsion (Louis et al., 2008a; Asahina et al., 2009). Rather, the data suggest that the saturation of olfactory input is preventing the larvae from responding to a decrease in odor concentration as they navigate away from the odor drop.

\section{Odd neurons likely boost olfactory input}

This would imply that the Odd neurons are not the only pathway controlling chemotaxis. It is feasible that a separate neural pathway controls odor navigation at high concentrations and the Odd neurons would then be the dedicated pathway for transmitting low concentrations of odor stimuli. However, the model that is most consistent with our data proposes that the Odd neurons function to boost sensory input to enhance signal detection in the olfactory system. Several neural systems incorporate background activity to boost faint signals above threshold (Wiesenfeld and Moss, 1995). Local excitatory interneurons in the adult Drosophila antennal lobe redistribute odor-evoked activity in PNs (Shang et al., 2007), which could result in a boost of PN activity when odor concentrations are low. There are several similarities between excitatory interneurons in the adult AL and the larval Odd neurons. The Odd neurons are also excitatory and respond to a variety of complex and monomolecular odors (Fig. 5). Excitatory interneurons in the AL have not been studied in the larvae, and it is not known whether they play a similar role as in the adult. It could be that larvae have adopted an alternative strategy to boost the transmission of low concentrations of odor stimuli through the Odd neurons. Boosting sensory input would be particularly important during foraging in conditions where odor stimuli are low further away from the food source.

A validation of this hypothesis would require a functional description of the neurons postsynaptic to the Odd neurons. Presently, this pathway is not known. Our descriptive study of the Odd neural anatomy and the functional study of PN activity in larvae in which the Odd neural function is modulated show that they do not connect directly with motor neurons or provide feedback to the AL. Thus, we can only speculate about the circuit postsynaptic to the Odd neurons. In Figure 10, we have attempted to place the Odd neurons within an olfactory circuit diagram. We show that Odd neurons receive direct input from
PNs with a possible short-term memory contribution via the Kenyon cells.

We speculate that the Odd neurons in turn synapse onto a downstream neural pathway that likely is also involved in chemotaxis. The existence of such a pathway is supported by our data, since modulating Odd neural function does not abolish chemotaxis but simply impair navigation. This strongly argues for a second system working a parallel with the Odd neurons controlling odor tracking. If this is the case, then the downstream pathway should also synapse onto the olfactory system, likely receiving direct input from the PNs. Innate behavior such as chemotaxis is thought to be associated with the lateral horn, and it is tempting to speculate that the pathway postsynaptic to the Odd neurons receives odor information from $\mathrm{PN}$ axons in the lateral horn. Excitatory input from the Odd neurons onto this pathway would then boost their activity, allowing these neurons to respond to low odor stimulus.

Thus, we have identified a distinct higher-order olfactory pathway dedicated to navigation during chemotaxis, providing us with a framework to begin to understand how animals can navigate within an odor gradient.

\section{References}

Akerboom J, Chen TW, Wardill TJ, Tian L, Marvin JS, Mutlu S, Calderón NC, Esposti F, Borghuis BG, Sun XR, Gordus A, Orger MB, Portugues R, Engert F, Macklin JJ, Filosa A, Aggarwal A, Kerr RA, Takagi R, Kracun S, et al (2012) Optimization of a GCaMP calcium indicator for neural activity imaging. J Neurosci 32:13819-13840. CrossRef Medline

Asahina K, Louis M, Piccinotti S, Vosshall LB (2009) A circuit supporting concentration-invariant odor perception in Drosophila. J Biol 8:9. CrossRef Medline

Baek JH, Cosman P, Feng Z, Silver J, Schafer WR (2002) Using machine vision to analyze and classify Caenorhabditis elegans behavioral phenotypes quantitatively. J Neurosci Methods 118:9-21. CrossRef Medline

Baines RA, Uhler JP, Thompson A, Sweeney ST, Bate M (2001) Altered electrical properties in Drosophila neurons developing without synaptic transmission. J Neurosci 21:1523-1531. Medline

Benton R (2009) Molecular basis of odor detection in insects. Ann N Y Acad Sci 1170:478-481. CrossRef Medline

Bras-Pereira C, Bessa J, Casares F (2006) Odd-skipped genes specify the signaling center that triggers retinogenesis in Drosophila. Development 133:4145-4149. CrossRef Medline

Burke CJ, Huetteroth W, Owald D, Perisse E, Krashes MJ, Das G, Gohl D, Silies M, Certel S, Waddell S (2012) Layered reward signalling through octopamine and dopamine in Drosophila. Nature 492:433-437. CrossRef Medline

Chen TW, Wardill TJ, Sun Y, Pulver SR, Renninger SL, Baohan A, Schreiter ER, Kerr RA, Orger MB, Jayaraman V, Looger LL, Svoboda K, Kim DS (2013) Ultrasensitive fluorescent proteins for imaging neuronal activity. Nature 499:295-300. CrossRef Medline

Christiansen F, Zube C, Andlauer TF, Wichmann C, Fouquet W, Owald D, Mertel S, Leiss F, Tavosanis G, Luna AJ, Fiala A, Sigrist SJ (2011) Presynapses in Kenyon cell dendrites in the mushroom body calyx of Drosophila. J Neurosci 31:9696-9707. CrossRef Medline

Cobb M, Domain I (2000) Olfactory coding in a simple system: adaptation in Drosophila larvae. Proc Biol Sci 267:2119-2125. CrossRef Medline

Cognigni P, Bailey AP, Miguel-Aliaga I (2011) Enteric neurons and systemic signals couple nutritional and reproductive status with intestinal homeostasis. Cell Metab 13:92-104. CrossRef Medline

Cole SH, Carney GE, McClung CA, Willard SS, Taylor BJ, Hirsh J (2005) Two functional but noncomplementing Drosophila tyrosine decarboxylase genes: distinct roles for neural tyramine and octopamine in female fertility. J Biol Chem 280:14948-14955. CrossRef Medline

Daniels RW, Gelfand MV, Collins CA, DiAntonio A (2008) Visualizing glutamatergic cell bodies and synapses in Drosophila larval and adult CNS. J Comp Neurol 508:131-152. CrossRef Medline

Davis RL (1993) Mushroom bodies and Drosophila learning. Neuron 11:1-14. CrossRef Medline

Fishilevich E, Domingos AI, Asahina K, Naef F, Vosshall LB, Louis M (2005) 
Chemotaxis behavior mediated by single larval olfactory neurons in Drosophila. Curr Biol 15:2086-2096. CrossRef Medline

Gershow M, Berck M, Mathew D, Luo L, Kane EA, Carlson JR, Samuel AD (2012) Controlling airborne cues to study small animal navigation. Nature methods 9:290-296. CrossRef Medline

Gomez-Marin A, Louis M (2014) Multilevel control of run orientation in Drosophila larval chemotaxis. Front Behav Neurosci 8:38. CrossRef Medline

Gomez-Marin A, Stephens GJ, Louis M (2011) Active sampling and decision making in Drosophila chemotaxis. Nat Commun 2:441. CrossRef Medline

Gordon MD, Scott K (2009) Motor control in a Drosophila taste circuit. Neuron 61:373-384. CrossRef Medline

Haag J, Borst A (2002) Dendro-dendritic interactions between motionsensitive large-field neurons in the fly. J Neurosci 22:3227-3233. Medline

Hallem EA, Carlson JR (2004) The odor coding system of Drosophila. Trends Genet 20:453-459. CrossRef Medline

Hao I, Green RB, Dunaevsky O, Lengyel JA, Rauskolb C (2003) The oddskipped family of zinc finger genes promotes Drosophila leg segmentation. Dev Biol 263:282-295. CrossRef Medline

Heimbeck G, Bugnon V, Gendre N, Keller A, Stocker RF (2001) A central neural circuit for experience-independent olfactory and courtship behavior in Drosophila melanogaster. Proc Natl Acad Sci U S A 98:1533615341. CrossRef Medline

Johard HA, Enell LE, Gustafsson E, Trifilieff P, Veenstra JA, Nässel DR (2008) Intrinsic neurons of Drosophila mushroom bodies express short neuropeptide F: relations to extrinsic neurons expressing different neurotransmitters. J Comp Neurol 507:1479-1496. CrossRef Medline

Kreher SA, Mathew D, Kim J, Carlson JR (2008) Translation of sensory input into behavioral output via an olfactory system. Neuron 59:110-124. CrossRef Medline

Larsen C, Franch-Marro X, Hartenstein V, Alexandre C, Vincent JP (2006) An efficient promoter trap for detection of patterned gene expression and subsequent functional analysis in Drosophila. Proc Natl Acad Sci U S A 103:17813-17817. CrossRef Medline

Lee T, Luo L (2001) Mosaic analysis with a repressible cell marker (MARCM) for Drosophila neural development. Trends Neurosci 24:251254. CrossRef Medline

Levy P, Larsen C (2013) Odd-skipped labels a group of distinct neurons associated with the mushroom body and optic lobe in the adult Drosophila brain. J Comp Neurol 521:3716-3740. CrossRef Medline

Li H, Chaney S, Roberts IJ, Forte M, Hirsh J (2000) Ectopic G-protein expression in dopamine and serotonin neurons blocks cocaine sensitization in Drosophila melanogaster. Curr Biol 10:211-214. CrossRef Medline

Louis M, Piccinotti S, Vosshall LB (2008a) High-resolution measurement of odor-driven behavior in Drosophila larvae. J Vis Exp pii:638. CrossRef Medline

Louis M, Huber T, Benton R, Sakmar TP, Vosshall LB (2008b) Bilateral olfactory sensory input enhances chemotaxis behavior. Nat Neurosci 11: 187-199. CrossRef Medline

Masuda-Nakagawa LM, Gendre N, O'Kane CJ, Stocker RF (2009) Localized olfactory representation in mushroom bodies of Drosophila larvae. Proc Natl Acad Sci U S A 106:10314-10319. CrossRef Medline

Mishra D, Chen YC, Yarali A, Oguz T, Gerber B (2013) Olfactory memories are intensity specific in larval Drosophila. J Exp Biol 216:1552-1560. CrossRef Medline

Nicolaï LJ, Ramaekers A, Raemaekers T, Drozdzecki A, Mauss AS, Yan J, Landgraf M, Annaert W, Hassan BA (2010) Genetically encoded dendritic marker sheds light on neuronal connectivity in Drosophila. Proc Natl Acad Sci U S A 107:20553-20558. CrossRef Medline

Nitabach MN, Sheeba V, Vera DA, Blau J, Holmes TC (2005) Membrane electrical excitability is necessary for the free-running larval Drosophila circadian clock. J Neurobiol 62:1-13. CrossRef Medline

Nitabach MN, Wu Y, Sheeba V, Lemon WC, Strumbos J, Zelensky PK, White BH, Holmes TC (2006) Electrical hyperexcitation of lateral ventral pacemaker neurons desynchronizes downstream circadian oscillators in the fly circadian circuit and induces multiple behavioral periods. J Neurosci 26:479-489. CrossRef Medline

Pereanu W, Kumar A, Jennett A, Reichert H, Hartenstein V (2010) Development-based compartmentalization of the Drosophila central brain. J Comp Neurol 518:2996-3023. CrossRef Medline

Pfeiffer BD, Ngo TT, Hibbard KL, Murphy C, Jenett A, Truman JW, Rubin GM (2010) Refinement of tools for targeted gene expression in Drosophila. Genetics 186:735-755. CrossRef Medline

Ramaekers A, Magnenat E, Marin EC, Gendre N, Jefferis GS, Luo L, Stocker RF (2005) Glomerular maps without cellular redundancy at successive levels of the Drosophila larval olfactory circuit. Curr Biol 15:982-992. CrossRef Medline

Risse B, Thomas S, Otto N, Löpmeier T, Valkov D, Jiang X, Klämbt C (2013) FIM, a novel FTIR-based imaging method for high throughput locomotion analysis. PLoS One 8:e53963. CrossRef Medline

Rosenzweig M, Brennan KM, Tayler TD, Phelps PO, Patapoutian A, Garrity PA (2005) The Drosophila ortholog of vertebrate TRPA1 regulates thermotaxis. Genes Dev 19:419-424. CrossRef Medline

Salvaterra PM, Kitamoto T (2001) Drosophila cholinergic neurons and processes visualized with Gal4/UAS-GFP. Brain Res Gene Expr Patterns 1:73-82. CrossRef Medline

Shang Y, Claridge-Chang A, Sjulson L, Pypaert M, Miesenböck G (2007) Excitatory local circuits and their implications for olfactory processing in the fly antennal lobe. Cell 128:601-612. CrossRef Medline

Sudhakaran IP, Holohan EE, Osman S, Rodrigues V, Vijayraghavan K, Ramaswami M (2012) Plasticity of recurrent inhibition in the Drosophila antennal lobe. J Neurosci 32:7225-7231. CrossRef Medline

Wiesenfeld K, Moss F (1995) Stochastic resonance and the benefits of noise: from ice ages to crayfish and SQUIDs. Nature 373:33-36. CrossRef Medline

Zhang YQ, Rodesch CK, Broadie K (2002) Living synaptic vesicle marker: synaptotagmin-GFP. Genesis 34:142-145. CrossRef Medline

Zheng Y, Wildonger J, Ye B, Zhang Y, Kita A, Younger SH, Zimmerman S, Jan LY, Jan YN (2008) Dynein is required for polarized dendritic transport and uniform microtubule orientation in axons. Nat Cell Biol 10:11721180. CrossRef Medline 